\title{
HYPERELLIPTIC $d$-OSCULATING COVERS AND RATIONAL SURFACES
}

\author{
ARMANDO TREIBICH
}

\section{INTRODUCTION}

1.1. Let $\mathbb{P}^{1}$ and $(X, q)$ denote, respectively, the projective line and a fixed elliptic curve marked at its origin, both defined over an algebraically closed field $\mathbb{K}$ of arbitrary characteristic $\boldsymbol{p} \neq 2$. We will study all finite separable marked morphisms $\pi:(\Gamma, p) \rightarrow(X, q)$, called hereafter hyperelliptic covers, such that $\Gamma$ is a degree- 2 cover of $\mathbb{P}^{1}$, ramified at the smooth point $p \in \Gamma$. Canonically associated to $\pi$ there is the Abel (rational) embedding of $\Gamma$ into its generalized Jacobian, $A_{p}: \Gamma \rightarrow J a c \Gamma$, and $\{0\} \subsetneq V_{\Gamma, p}^{1} \ldots \subsetneq V_{\Gamma, p}^{g}$, the flag of hyperosculating planes to $A_{p}(\Gamma)$ at $A_{p}(p) \in J a c \Gamma$ (cf. 2.1. \& 2.2.). On the other hand, we also have the homomorphism $\iota_{\pi}: X \rightarrow$ Jac $\Gamma$, obtained by dualizing $\pi$. There is a smallest positive integer $d$ such that the tangent line to $\iota_{\pi}(X)$ is contained in the $d$-dimensional osculating plane $V_{\Gamma, p}^{d}$. We call it the osculating order of $\pi$, and $\pi$ a hyperelliptic $d$-osculating cover (2.4.(2)). If $\pi$ factors through another hyperelliptic cover, the arithmetic genus increases, while the osculating order can not decrease (2.8.).

Studying, characterizing and constructing those with given osculating order $d$ but maximal possible arithmetic genus, so-called minimal-hyperelliptic d-osculating covers, will be one of the main issues of this article. The other one, to which the first issue reduces, is the construction of all rational curves in a particular anticanonical rational surface associated to $X$ (i.e.: a rational surface with an effective anticanonical divisor). Both problems are interesting on their own and in any characteristic $\boldsymbol{p} \neq 2$. They were first considered, however, over the complex numbers and through their link with solutions of the Korteweg-deVries hierarchy, doubly periodic with respect to the $d$-th $K d V$ flow (cf. 11, 3], 8, , 9, 14 for $d=1$ and 11, 2, 44, 5] for $d=2$ ). We sketch hereafter the structure and main results of our article.

(1) We start defining in section 2. the Abel rational embedding $A_{p}: \Gamma \rightarrow J a c \Gamma$, and construct the flag $\{0\} \subsetneq V_{\Gamma, p}^{1} \ldots \subsetneq V_{\Gamma, p}^{g}=H^{1}\left(\Gamma, O_{\Gamma}\right)$, of hyperosculating planes at the image of any smooth point $p \in \Gamma$. We then define the homomorphism $\iota_{\pi}: X \rightarrow J a c \Gamma$, canonically associated to the hyperelliptic cover $\pi$, and its osculating order (2.4.(2)). Regardless of the osculating order, we prove that any degree- $n$ hyperelliptic cover has odd ramification index at the marked point, say $\rho$, and factors through a unique one of maximal arithmetic genus $2 n-\frac{\rho+1}{2}(\mathbf{2 . 6}$.). We finish characterizing the osculating order by the existence of a particular projection $\kappa: \Gamma \rightarrow \mathbb{P}^{1}(\mathbf{2 . 6}$.).

(2) The $d$-osculating criterion 2.6. paves the way to the algebraic surface approach developed in the remaining sections. The main characters are 
played by (two morphisms between) three projective surfaces, canonically associated to the elliptic curve $X$ :

- $e: S^{\perp} \rightarrow S$ : the blowing-up of a particular ruled surface $\pi_{S}: S \rightarrow X$, at the 8 fixed points of its involution;

- $\varphi: S^{\perp} \rightarrow \widetilde{S}:$ a projection onto an anticanonical rational surface.

(3) Once $S, S^{\perp}$ and $\widetilde{S}$ are constructed (3.2., 3.4.), we prove that any hyperelliptic d-osculating cover $\pi:(\Gamma, p) \rightarrow(X, q)$ factors canonically through a curve $\Gamma^{\perp} \subset S^{\perp}$, and projects, via $\varphi: S^{\perp} \rightarrow \widetilde{S}$, onto a rational irreducible curve $\widetilde{\Gamma} \subset \widetilde{S}$ (3.8.). We also prove that any hyperelliptic d-osculating cover dominates a unique one of same osculating order $d$, but maximal arithmetic genus, so-called minimal-hyperelliptic (3.9.). Conversely, given $\widetilde{\Gamma} \subset \widetilde{S}$, we study when and how one can recover all minimal-hyperelliptic d-osculating covers having same canonical projection $\widetilde{\Gamma}(\mathbf{3 . 1 1}$.) .

(4) Section 4. is mainly devoted to studying the linear equivalence class of the curve $\Gamma^{\perp} \subset S^{\perp}$, canonically associated to any hyperelliptic $d$-osculating cover $\pi$, and associated invariants (4.3. \& 4.4.). We end up with a numerical characterization of minimal-hyperelliptic d-osculating covers (4.6.).

(5) At last, we dress the list of all (-1) and (-2)-irreducible curves of $\widetilde{S}$ (5.7.), needed to study its nef cone, and give, for any $n, d \in \mathbb{N}^{*}$, two different constructions of $(d-1)$-dimensional families of smooth, degree- $n$, minimalhyperelliptic d-osculating covers: one based on Brian Harbourne's results on anticanonical rational surfaces ([6]), the other one based on [13] and leading, ultimately, to explicit equations for the corresponding covers.

\section{JACOBIANS OF CURVES AND HYPERELLIPTIC $d$-OSCULATING COVERS}

2.1. Let $\mathbb{K}$ be an algebraically closed field of characteristic $\boldsymbol{p} \neq 2, \mathbb{P}^{1}$ the projective line over $\mathbb{K}$ and $(X, q)$ a fixed elliptic curve, also defined over $\mathbb{K}$. The latter will be equipped with its canonical symmetry $[-1]:(X, q) \rightarrow(X, q)$, fixing $\omega_{o}:=q$, as well as the other three half-periods $\left\{\omega_{j}, j=1,2,3\right\}$. We will also choose once for all, an odd local parameter of $X$ centered at $q$, say $z$, such that $z \circ[-1]=-z$.

By a curve we will mean hereafter a complete integral curve over $\mathbb{K}$, say $\Gamma$, of positive arithmetic genus $g>0$. The moduli space of degree- 0 invertible sheaves over $\Gamma$, denoted by $J a c \Gamma$ and called the generalized Jacobian of $\Gamma$, is a $g$-dimensional connected commutative algebraic group, canonically identified to $H^{1}\left(\Gamma, O_{\Gamma}^{*}\right)$, with tangent space at its origin equal to $H^{1}\left(\Gamma, O_{\Gamma}\right)$. Recall also the Abel (rational) embedding $A_{p}: \Gamma \rightarrow J a c \Gamma$, sending any smooth point $p^{\prime} \in \Gamma$ to the isomorphism class of $O_{\Gamma}\left(p^{\prime}-p\right)$. For any marked curve $(\Gamma, p)$ as above, and any positive integer $j$, let us consider the exact sequence of $O_{\Gamma}$-modules $0 \rightarrow O_{\Gamma} \rightarrow O_{\Gamma}(j p) \rightarrow O_{j p}(j p) \rightarrow 0$, as well as the corresponding long exact cohomology sequence :

$$
0 \rightarrow H^{0}\left(\Gamma, O_{\Gamma}\right) \rightarrow H^{0}\left(\Gamma, O_{\Gamma}(j p)\right) \rightarrow H^{0}\left(\Gamma, O_{j p}(j p)\right) \stackrel{\delta}{\rightarrow} H^{1}\left(\Gamma, O_{\Gamma}\right) \rightarrow \ldots
$$


where $\delta: H^{0}\left(\Gamma, O_{j p}(j p)\right) \rightarrow H^{1}\left(\Gamma, O_{\Gamma}\right)$ is the cobord morphism. According to the Weierstrass gap Theorem, for any $d \in\{1, \ldots, g\}$, there exists $0<j<2 g$ such that $\delta\left(H^{0}\left(\Gamma, O_{j p}(j p)\right)\right)$ is a $d$-dimensional subpace, denoted hereafter by $V_{\Gamma, p}^{d}$.

For a generic point $p$ of $\Gamma$ we have $V_{\Gamma, p}^{d}=\delta\left(H^{0}\left(\Gamma, O_{d p}(d p)\right)\right)(i . e .: j=d)$, while for any $p \in \Gamma$, the tangent to $A_{p}(\Gamma)$ at 0 is equal to $V_{\Gamma, p}^{1}=\delta\left(H^{0}\left(\Gamma, O_{p}(p)\right)\right)$.

\section{Definition 2.2.}

(1) The filtration $\{0\} \subsetneq V_{\Gamma, p}^{1} \ldots \subsetneq V_{\Gamma, p}^{g}=H^{1}\left(\Gamma, O_{\Gamma}\right)$ will be called the flag of hyperosculating spaces to $A_{p}(\Gamma)$ at 0 .

(2) The curve $\Gamma$ will be called a hyperelliptic curve, and $p \in \Gamma$ a Weierstrass point, if there exists a degree-2 projection onto $\mathbb{P}^{1}$, ramified at $p$. Or equivalently, if there exists an involution, denoted in the sequel by $\tau_{\Gamma}: \Gamma \rightarrow \Gamma$ and called the hyperelliptic involution, fixing $p$ and such that the quotient curve $\Gamma / \tau_{\Gamma}$ is isomorphic to $\mathbb{P}^{1}$.

Proposition 2.3. $(12 \S 1.6$.

Let $(\Gamma, p, \lambda)$ be a hyperelliptic curve of arithmetic genus $g$, equipped with a local parameter $\lambda$, centered at a smooth Weierstrass point $p \in \Gamma$. For any odd integer $1 \leq j:=2 d-1 \leq g$, consider the exact sequence of $O_{\Gamma}$-modules:

$$
0 \rightarrow O_{\Gamma} \rightarrow O_{\Gamma}(j p) \rightarrow O_{j p}(j p) \rightarrow 0
$$

as well as its long exact cohomology sequence

$$
0 \rightarrow H^{0}\left(\Gamma, O_{\Gamma}\right) \rightarrow H^{0}\left(\Gamma, O_{\Gamma}(j p)\right) \rightarrow H^{0}\left(\Gamma, O_{j p}(j p)\right) \stackrel{\delta}{\rightarrow} H^{1}\left(\Gamma, O_{\Gamma}\right) \rightarrow \ldots,
$$

$\delta$ being the cobord morphism.

For any, $m \geq 1$, we also let $\left[\lambda^{-m}\right]$ denote the class of $\lambda^{-m}$ in $H^{0}\left(\Gamma, O_{m p}(m p)\right)$. Then $V_{\Gamma, p}^{d}$ is generated by $\left\{\delta\left(\left[\lambda^{2 l-1}\right]\right), l=1, . ., d\right\}$. In other words, the d-th osculating subspace to $A_{p}(\Gamma)$ at 0 is equal to $\delta\left(H^{0}\left(\Gamma, O_{j p}(j p)\right)\right)$, for $j=2 d-1$.

\section{Definition 2.4.}

(1) A finite separable marked morphism $\pi:(\Gamma, p) \rightarrow(X, q)$, such that $\Gamma$ is a hyperelliptic curve and $p \in \Gamma$ a smooth Weierstrass point, will be called a hyperelliptic cover. We will say that $\pi$ dominates another hyperelliptic cover $\bar{\pi}:(\bar{\Gamma}, \bar{p}) \rightarrow(X, q)$, if there exists a degree-1 morphism $j:(\Gamma, p) \rightarrow(\bar{\Gamma}, \bar{p})$, such that $\pi=\bar{\pi} \circ j$.

(2) Let $\iota_{\pi}: X \rightarrow J a c \Gamma$ denote the group homomorphism $q^{\prime} \mapsto A_{p}\left(\pi^{*}\left(q^{\prime}-q\right)\right)$. There is a minimal integer $d \geq 1$, called henceforth osculating order of $\pi$, such that the tangent to $\iota_{\pi}(X)$ at 0 is contained in $V_{\Gamma, p}^{d}$. We will then call $\pi$ a hyperelliptic $d$-osculating cover.

\section{Proposition 2.5.}

Let $\pi:(\Gamma, p) \rightarrow(X, q)$ be a degree-n hyperelliptic cover with ramification index $\rho$ at $p, f:(\Gamma, p) \rightarrow\left(\mathbb{P}^{1}, \infty\right)$ the corresponding degree-2 projection, ramified at $p$, and let $\Gamma_{f, \pi}$ denote the image curve $(f, \pi)(\Gamma) \subset \mathbb{P}^{1} \times X$. Then (see diagram below), 
(1) the hyperelliptic involution $\tau_{\Gamma}$ satisfies $[-1] \circ \pi=\pi \circ \tau_{\Gamma}$ and $\rho$ is odd;

(2) $\Gamma_{f, \pi}$ has arithmetic genus $2 n-1$ and is unibranch at $(\infty, q)$;

(3) let $(\bar{\Gamma}, \bar{p})$ denote the partial desingularization of $\Gamma_{f, \pi}$ at $(\infty, q)$, equipped with its canonical projection via $\Gamma_{f, \pi}$, say $\bar{\pi}:(\bar{\Gamma}, \bar{p}) \rightarrow(X, q)$, then:

$\bar{\pi}$ is a hyperelliptic cover of arithmetic genus $2 n-\frac{1}{2}(\rho+1)$;

(4) $\pi$, as well as any hyperelliptic cover dominated by $\pi$, factors through $\bar{\pi}$.

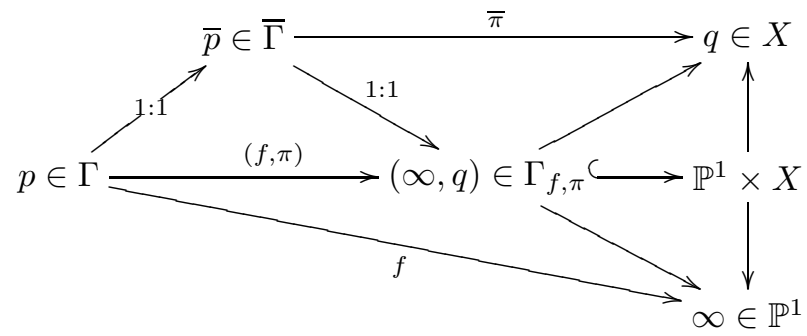

Proof. (1) Let $A l b_{\pi}: J a c \Gamma \rightarrow J a c X$ denote the Albanese homomorphism, sending any $L \in J a c \Gamma$ to $A l b_{\pi}(L):=\operatorname{det}\left(\pi_{*} L\right) \otimes \operatorname{det}\left(\pi_{*} O_{\Gamma}\right)^{-1}$, and $\Gamma^{0}$ denote the open dense subset of smooth points of $\Gamma$. Up to identifying $\operatorname{Jac} X$ with $(X, q)$, we know that $A l b_{\pi} \circ \iota_{\pi}=[n]$, the multiplication by $n$, and $A l b_{\pi} \circ A_{p}$ is well defined over $\Gamma^{0}$ and equal there to $\pi$. Knowing, on the other hand, that $A_{p} \circ \tau_{\Gamma}=[-1] \circ A_{p}$, we deduce that $\pi \circ \tau_{\Gamma}=A l b_{\pi} \circ A_{p} \circ \tau_{\Gamma}=[-1] \circ A l b_{\pi} \circ A_{p}=[-1] \circ \pi$ (over the open dense subset $\Gamma^{0}$, hence) over all $\Gamma$ as asserted.

(2) \& (3) The projections $f$ and $\pi$ have degrees 2 and $n$, implying that $\Gamma_{f, \pi}$ is numerically equivalent to $n \cdot\{\infty\} \times X+2 . \mathbb{P}^{1} \times\{q\}$ and, by means of the adjunction formula, that it has arithmetic genus $2 n-1$. We also know that $f$ and $\pi$ have ramification indices 2 and $\rho$ at $p \in \Gamma$. Hence, $\Gamma_{f, \pi}$ intersects the fibers $\mathbb{P}^{1} \times\{q\}$ and $\{\infty\} \times X$ at $(\infty, q)$, with multiplicities $\rho$ and 2. Adding property 2.5.(1) we deduce that its local equation at $(\infty, q)$ can only have even powers of $z$, and must be equal to $z^{2}=w^{\rho} h\left(w, z^{2}\right)$, for some invertible element $h$ (i.e.: $\left.h(0,0) \neq 0\right)$. In particular $\Gamma_{f, \pi}$ is unibranch and has multiplicity $\min \{2, \rho\}$ at $(\infty, q)$. Moreover, for its desingularization over $(\infty, q), \frac{\rho-1}{2}$ successive monoidal transformation are necessary, each one of which decreases the arithmetic genus by 1 . Hence $\bar{\Gamma}$ has arithmetic genus $2 n-1-\frac{\rho-1}{2}=2 n-\frac{\rho+1}{2}$ as asserted.

(4) Since $\Gamma$ is already smooth at $p$, we immediately see that $(f, \pi)$ factors through $\bar{\pi}$. Hence, $\pi$ dominates $\bar{\pi}$ as asserted. Reciprocally, any other hyperelliptic cover dominated by $\pi$ must factor through $\left(\Gamma_{f, \pi},(\infty, q)\right)$, and should lift to its partial desingularization $(\bar{\Gamma}, \bar{p})$. In other words, it should dominate $\bar{\pi}$.

\section{Theorem 2.6.}

The osculating order of an hyperelliptic cover $\pi:(\Gamma, p) \rightarrow(X, q)$, is the minimal integer $d \geq 1$ for which there exists a morphism $\kappa: \Gamma \rightarrow \mathbb{P}^{1}$ satisfying :

(1) the poles of $\kappa$ lie along $\pi^{-1}(q)$;

(2) $\kappa+\pi^{*}\left(z^{-1}\right)$ has a pole of order $2 d-1$ at $p$, and no other pole along $\pi^{-1}(q)(\mathbf{2 . 1 .})$. 
Furthermore, for such $d$ there exists a unique morphism $\kappa: \Gamma \rightarrow \mathbb{P}^{1}$ satisfying properties (1)\&(2) above, as well as (2.2.(2)):

(3) $\tau_{\Gamma}^{*}(\kappa)=-\kappa$.

Proof. According to 2.3., $\forall r \geq 1$ the $r$-th osculating subspace $V_{\Gamma, p}^{r}$ is generated by $\left\{\delta\left(\left[\lambda^{-(2 l-1)}\right]\right), l=1, . ., r\right\}$. On the other hand, $\pi$ being separable, the tangent to $\iota_{\pi}(X) \subset J a c \Gamma$ at 0 is equal to $\pi^{*}\left(H^{1}\left(X, O_{X}\right)\right)$, hence, generated by $\delta\left(\left[\pi^{*}\left(z^{-1}\right)\right]\right)$.

In other words, the osculating order $d$ is the smallest positive integer such that $\delta\left(\left[\pi^{*}\left(z^{-1}\right)\right]\right)$ is a linear combination $\sum_{l=1}^{d} a_{l} \delta\left(\left[\lambda^{-(2 l-1)}\right]\right)$, with $a_{d} \neq 0$. Or equivalently, thanks to the Mittag-Leffler Theorem, the smallest for which there exists a morphism $\kappa: \Gamma \rightarrow \mathbb{P}^{1}$, with polar parts equal to $\pi^{*}\left(z^{-1}\right)-\sum_{l=1}^{d} a_{l} \lambda^{-(2 l-1)}$. The latter conditions on $\kappa$ are equivalent to 2.6.(1) \& (2). Moreover, up to replacing $\kappa$ by $\frac{1}{2}\left(\kappa-\tau_{\Gamma}^{*}(\kappa)\right)$, we can assume $\kappa$ is $\tau_{\Gamma}$-anti-invariant. The difference of two such functions should be $\tau_{\Gamma}$-anti-invariant, while having a unique pole at $p$, of order strictly smaller than $2 d-1 \leq 2 g-1$, where $g$ denotes the arithmetic genus of $\Gamma$. Hence the difference is identically zero, implying the uniqueness of such a morphism $\kappa$.

\section{Definition 2.7.}

(1) The pair of marked projections $(\pi, \kappa)$, satisfying 2.6.(1),(2)\&(3), will be called a hyperelliptic d-osculating pair, and $\kappa$ the hyperelliptic $d$-osculating function associated to $\pi$.

(2) If the latter $\pi:(\Gamma, p) \rightarrow(X, q)$ does not dominate any other hyperelliptic $d$-osculating cover, we will call it minimal-hyperelliptic d-osculating cover.

\section{Corollary 2.8.}

Let $\pi:(\Gamma, p) \rightarrow(X, q)$ and $\pi^{\prime}:\left(\Gamma^{\prime}, p\right) \rightarrow(X, q)$ be two hyperelliptic covers of osculating orders, $d$ and $d^{\prime}$ respectively, such that $\pi$ dominates $\pi^{\prime}$. Then $d \leq d^{\prime}$.

Proof. Let $\kappa^{\prime}$ be the hyperelliptic d-osculating function associated to $\pi^{\prime}$, and $j:(\Gamma, p) \rightarrow\left(\Gamma^{\prime}, p^{\prime}\right)$ the birational morphism such that $\pi=\pi^{\prime} \circ j$. Then, the poles of $\kappa^{\prime} \circ j: \Gamma \rightarrow \mathbb{P}^{1}$ lie along $\pi^{-1}(q)$, while $\kappa^{\prime} \circ j+\pi^{*}\left(z^{-1}\right)=\left(\kappa^{\prime}+\pi^{\prime *}\left(z^{-1}\right)\right) \circ j$ has a pole of order $2 d^{\prime}-1$ and no other pole along $\pi^{-1}(q)$. It follows (along the same lines of proof as in 2.6.) that the tangent to $\iota_{\pi}(X)$ must be contained in $V_{\Gamma, p}^{d^{\prime}}$. Hence, the minimality of $d$ implies $d \leq d^{\prime}$

\section{The Algebraic surface SET UP}

3.1. We will construct hereafter the ruled surface $\pi_{S}: S \rightarrow X$ and its blowing-up $e: S^{\perp} \rightarrow S$, both naturally equipped with involutions $\tau: S \rightarrow S$ and $\tau^{\perp}: S^{\perp} \rightarrow S^{\perp}$, as well as a degree-2 projection $S^{\perp} \stackrel{\varphi}{\rightarrow} \widetilde{S}$ to a known anticanonical rational surface. We will then prove that any hyperelliptic d-osculating cover $\pi:(\Gamma, p) \rightarrow(X, q)$ factors uniquely through $\pi_{S^{\perp}}: \pi_{S} \circ e: S^{\perp} \rightarrow X$ and projects, via $S^{\perp} \rightarrow \widetilde{S}$, onto an irreducible rational curve. Moreover, we will prove that $\pi$ dominates a unique hyperelliptic d-osculating cover (3.9.).

\section{Definition 3.2.}

(1) Fix an odd meromorphic function $\zeta: X \rightarrow \mathbb{P}^{1}$, with divisor of zeroes and poles equal to $(\zeta)=q+\omega_{1}-\omega_{2}-\omega_{3}$, and consider the open affine subsets $U_{o}:=X \backslash\{q\}$ 
and $U_{1}:=X \backslash\left\{\omega_{1}\right\}$. We let $\pi_{S}: S \rightarrow X$ denote the ruled surface obtained by identifying $\mathbb{P}^{1} \times U_{o}$ with $\mathbb{P}^{1} \times U_{1}$, over $X \backslash\left\{q, \omega_{1}\right\}$ :

$$
\forall q^{\prime} \neq q, \omega_{1}, \quad\left(T_{o}, q^{\prime}\right) \in \mathbb{P}^{1} \times U_{o} \quad \text { is identified with } \quad\left(T_{1}+\frac{1}{\zeta\left(q^{\prime}\right)}, q^{\prime}\right) \in \mathbb{P}^{1} \times U_{1} .
$$

In other words, we glue the fibers of $\mathbb{P}^{1} \times U_{0}$ and $\mathbb{P}^{1} \times U_{1}$, over any $q^{\prime} \neq q, \omega_{1}$, by means of a translation. In particular the constant sections $q^{\prime} \in U_{k} \mapsto\left(\infty, q^{\prime}\right) \in$ $\mathbb{P}^{1} \times U_{k}(k=0,1)$, get glued together, defining a particular one denoted by $C_{o} \subset S$.

(2) The involutions $\mathbb{P}^{1} \times U_{k} \rightarrow \mathbb{P}^{1} \times U_{k}, \quad\left(T_{k}, q^{\prime}\right) \mapsto\left(-T_{k},[-1]\left(q^{\prime}\right)\right)(k=0,1)$, get glued under the above identification and define an involution $\tau: S \rightarrow S$, such that $\pi_{S} \circ \tau=[-1] \circ \pi_{S}$. In particular, $\tau$ has two fixed points over each half-period $\omega_{i}$ : one in $C_{o}$, denoted by $s_{i}$, and the other one denoted by $r_{i}(i=0, . ., 3)$. It can also be checked that translating along the fibers of $\mathbb{K} \times U_{k}$ by any scalar $a \in \mathbb{K}(k=0,1)$, extends to an automorphism $t_{a}: S \rightarrow S$, leaving fixed $C_{o}$ and such that $\pi_{S} \circ t_{a}=\pi_{S}$.

(3) Whenever $\boldsymbol{p} \geq 3$, we choose $\zeta$ (3.2.(1)) as a local parameter of $X$ centered at $q$, and consider the unique meromorphic function $f_{p}: X \rightarrow \mathbb{P}^{1}$, having a local development $f_{p}=\frac{1}{\zeta^{p}}+\frac{c}{\zeta}+O(\zeta)$, for some $c \in \mathbb{K}$. We denote $C_{p} \subset S$ the curve defined over $\mathbb{P}^{1} \times U_{o}$ by the equation $T_{o}^{p}+c T_{o}+f_{p}=0$, and over $\mathbb{P}^{1} \times U_{1}$ by the equation $T_{1}^{p}+c T_{1}+f_{p}-\frac{1}{\zeta^{p}}-\frac{c}{\zeta}=0$.

\section{Proposition 3.3.}

The ruled surface $S \rightarrow X$ has a unique section of self-intersection 0 , namely $C_{o}$, and its canonical divisor is equal to $-2 C_{o}$. In particular, $S \rightarrow X$ is isomorphic to $\mathbb{P}(E) \rightarrow X$, the ruled surface associated to the unique indecomposable rank-2, degree-0 vector bundle over $X(\mathrm{cf} .77 \S \mathrm{V} .2$, [14 $\S 3.1$.$) .$

Proof. The meromorphic differentials $d T_{o}$ and $d T_{1}$ get also glued together, implying that $K_{S}$, the canonical divisor of $S$ is represented by $-2 C_{o}$. Any section of $\pi_{S}: S \rightarrow X$, other than $C_{o}$, is given by two non-constant morphisms $f_{i}: U_{i} \rightarrow \mathbb{P}^{1}$ $(i=1,2)$, such that $f_{o}=f_{1}-\frac{1}{\zeta} \quad$ outside $\left\{q, \omega_{1}\right\}$. A straightforward calculation shows that a section as above intersects $C_{o}$, while having self-intersection number greater or equal to 2. It follows from the general Theory of Ruled Surfaces (cf. (7) $\S \mathrm{V} .2)$ that $C_{o}$ must be the unique section with zero self-intersection. Hence, the ruled surface $\pi_{S}: S \rightarrow X$ defined above, is isomorphic to the projectivization of the unique indecomposable rank-2, degree-0 vector bundle over $X(\mathrm{cf}$. $[7 \S \mathrm{V} .2)$.

Definition 3.4.(cf. [14 $\S 4.1$.

Let $e: S^{\perp} \rightarrow S$ denote hereafter the monoidal transformation of $S$ at $\left\{s_{i}, r_{i}, i=\right.$ $0, . ., 3\}$, the eight fixed points of $\tau$, and $\tau^{\perp}: S^{\perp} \rightarrow S^{\perp}$ its lift to an involution fixing the corresponding exceptional divisors $\left\{s_{i}^{\perp}:=e^{-1}\left(s_{i}\right), r_{i}^{\perp}:=e^{-1}\left(r_{i}\right), i=0, . ., 3\right\}$. Taking the quotient of $S^{\perp}$ with respect to $\tau^{\perp}$, we obtain a degree-2 projection $\varphi: S^{\perp} \rightarrow \widetilde{S}$, onto a smooth rational surface $\widetilde{S}$, ramified along the exceptional curves $\left\{s_{i}^{\perp}, r_{i}^{\perp}, i=0, . ., 3\right\}$.

\section{Lemma 3.5.}

Whenever $\boldsymbol{p} \geq 3$, the curve $C_{\boldsymbol{p}}$ (3.2.(3)) is irreducible and linearly equivalent to 
$p C_{o}$. Moreover, any irreducible curve numerically equivalent to a multiple of $C_{o}$, is either $C_{o}$ itself or a translate of $C_{p}$. In particular $C_{p}$ and $\boldsymbol{p} C_{o}$ generate the complete linear system $\left|p C_{o}\right|$, and $S$ is an elliptic surface.

Proof. The curve $C_{p}$ is $\tau$-invariant, does not intersect the section $C_{o}$ and projects onto $X$ with degree $\boldsymbol{p}$. Hence, $C_{\boldsymbol{p}}$ is linearly equivalent to $\boldsymbol{p} C_{o}$ and has multiplicity one at $r_{o} \in S$. In order to prove its irreducibility, we may assume $C_{p} \rightarrow X$ is separable, or equivalently, that $c \neq 0$ in 3.2.(3). Otherwise $C_{p} \rightarrow X$ would be purely inseparable and $C_{p}$ isomorphic to $X$. The curve $C_{p}$ is then smooth and transverse to the fiber $S_{o}:=\pi_{S}^{-1}(q)$, and their intersection number at $r_{o} \in S_{o} \cap C_{p}$ is equal to 1 . Let $C^{\prime}$ denote the unique irreducible $\tau$-invariant component of $C_{p}$ going through $r_{o}$, and suppose that $C^{\prime} \neq C_{p}$. Then $C^{\prime}$ has zero self-intersection and the projection $C^{\prime} \rightarrow X$ has odd degree $\boldsymbol{p}^{\prime}$, for some $1<\boldsymbol{p}^{\prime}<\boldsymbol{p}$. Otherwise (i.e.: if $\boldsymbol{p}^{\prime}=1$ ), $C^{\prime}$ would give another section of $\pi_{S}$ having zero self-intersection. Contradiction! Its complement, say $C^{\prime \prime}:=C_{p} \backslash C^{\prime}$, is a smooth, effective divisor linearly equivalent to $\left(\boldsymbol{p}-\boldsymbol{p}^{\prime}\right) C_{o}$. Translating $C^{\prime}$ by an appropiate automorphism $t_{a}$ (3.2.(2)), we may assume that $t_{a}\left(C^{\prime}\right)$ intersects $C^{\prime \prime}$, hence $t_{a}\left(C^{\prime}\right) \subset C^{\prime \prime}$ because their intersection number is equal to 0 . It follows that any irreducible component of $C_{p}$ is a translate of $C^{\prime}$, forcing the prime number $\boldsymbol{p}$ to be a multiple of $p^{\prime}>1$. Therefore, $\boldsymbol{p}=\boldsymbol{p}$ ' and $C_{\boldsymbol{p}}=C^{\prime}$ is irreducible as asserted. Consider at last, any other irreducible curve, say $C$, linearly equivalent to $m C_{o}$ for some $m>1$. It has zero intersection number with $C_{p}$ and must intersect some translate of $C_{p}$, implying that they coincide. In particular $m=\boldsymbol{p}$ and any element of $\left|\boldsymbol{p} C_{o}\right|$, other than $\boldsymbol{p} C_{o}$, is a translate of $C_{p}$.

The Lemma and Propositions hereafter, proved in $[12 \S 2.3 ., 22.4 ., \& \S 2.5$., will be instrumental in constructing the equivariant factorization $\iota^{\perp}: \Gamma \rightarrow S^{\perp}$ (3.2.).

\section{Lemma 3.6.}

There exists a unique, $\tau$-anti-invariant, rational morphism $\kappa_{s}: S \rightarrow \mathbb{P}^{1}$, with poles over $C_{o}+\pi_{S}^{-1}(q)$, such that over a suitable neighborhood $U$ of $q \in X$, the divisor of poles of $\kappa_{s}+\pi_{S}^{*}\left(z^{-1}\right)$ is reduced and equal to $C_{o} \cap \pi_{S}^{-1}(U)$.

\section{Proposition 3.7.}

For any hyperelliptic cover $\pi:(\Gamma, p) \rightarrow(X, q)$, the existence of the unique hyperelliptic d-osculating function $\kappa: \Gamma \rightarrow \mathbb{P}^{1}(\mathbf{2 . 7} \cdot(1))$ is equivalent to the existence of a unique morphism $\iota: \Gamma \rightarrow S$ such that $\iota \circ \tau_{\Gamma}=\tau \circ \iota, \pi=\pi_{S} \circ \iota$ and $\iota^{*}\left(C_{o}\right)=(2 d-1) p$.

\section{Proposition 3.8.}

For any hyperelliptic d-osculating pair $(\pi, \kappa)$, the above morphism $\iota: \Gamma \rightarrow S$ lifts to a unique equivariant morphism $\iota^{\perp}: \Gamma \rightarrow S^{\perp}$ (i.e.: $\left.\tau^{\perp} \circ \iota^{\perp}=\iota^{\perp} \circ \tau_{\Gamma}\right)$. In particular, $(\pi, \kappa)$ is the pullback of $\left(\pi_{S^{\perp}}, \kappa_{s^{\perp}}\right)=\left(\pi_{S} \circ e, \kappa_{s} \circ e\right)$, and $\Gamma$ lifts to a $\tau^{\perp}$-invariant curve, $\Gamma^{\perp}:=\iota^{\perp}(\Gamma) \subset S^{\perp}$, which projects onto the rational irreducible 
curve $\widetilde{\Gamma}:=\varphi\left(\Gamma^{\perp}\right) \subset \widetilde{S}$. In particular, $2 d-1=e^{*}\left(C_{o}\right) \cdot \iota^{\perp}{ }_{*}(\Gamma)$.

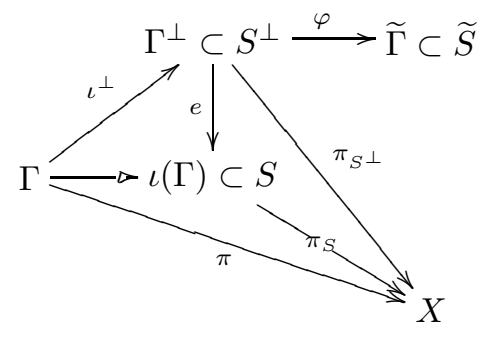

Proof. The monoidal transformation $e: S^{\perp} \rightarrow S$, as well as $\iota: \Gamma \rightarrow S$, can be pushed down to the corresponding quotients, making up the following diagram:

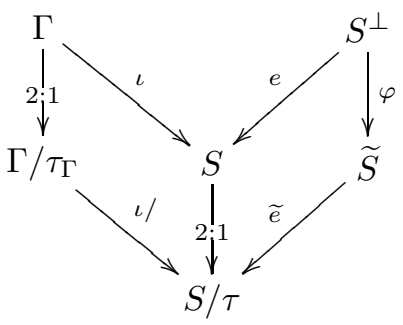

Moreover, since $\widetilde{e}: \widetilde{S} \rightarrow S / \tau$ is a birational morphism and $\Gamma / \tau_{\Gamma}$ is a smooth curve (in fact isomorphic to $\mathbb{P}^{1}$ ), we can lift $\iota /: \Gamma / \tau_{\Gamma} \rightarrow S / \tau$ to $\widetilde{S}$, obtaining a morphism $\widetilde{\iota}: \Gamma \rightarrow \widetilde{\Gamma} \subset \widetilde{S}$, fitting in the diagram:

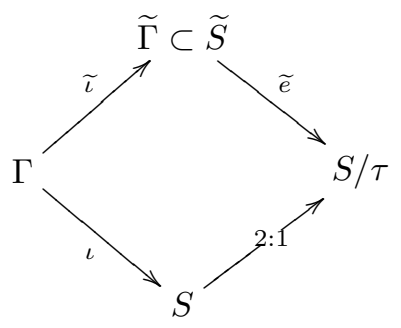

Recall now that $S^{\perp}$ is the fibre product of $\widetilde{e}: \widetilde{S} \rightarrow S / \tau$ and $S \rightarrow S / \tau$ (cf. 14] $\S 4.1$.). Hence, $\iota$ and $\widetilde{\iota}$ lift to a unique equivariant morphism $\iota^{\perp}: \Gamma \rightarrow \Gamma^{\perp} \subset S^{\perp}$, fitting in

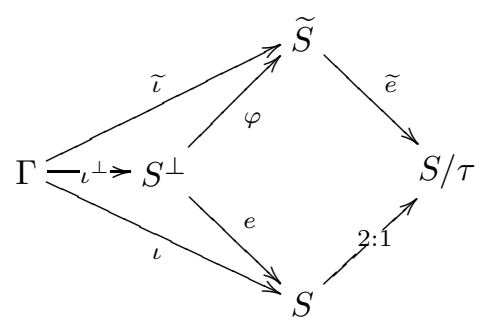

Furthermore, since $\widetilde{\iota}: \Gamma \rightarrow \widetilde{S}$ factors through $\Gamma \rightarrow \Gamma / \tau_{\Gamma} \cong \mathbb{P}^{1}$, its image $\widetilde{\Gamma}:=\varphi\left(\iota^{\perp}(\Gamma)\right)=\widetilde{\iota}(\Gamma) \subset \widetilde{S}$ is a rational irreducible curve as claimed. 


\section{Corollary 3.9.}

Any hyperelliptic d-osculating cover $\pi:(\Gamma, p) \rightarrow(X, q)$ dominates a unique minimalhyperelliptic d-osculating cover, with same image $\Gamma^{\perp} \subset S^{\perp}$ as $\pi$.

Proof. Let $\bar{\pi}:(\bar{\Gamma}, \bar{p}) \rightarrow(X, q)$ be an arbitrary hyperelliptic d-osculating cover dominated by $\pi:(\Gamma, p) \rightarrow(X, q), \psi:(\Gamma, p) \rightarrow(\bar{\Gamma}, \bar{p})$ the corresponding birational morphism and $\tau^{\perp}: \bar{\Gamma} \rightarrow S^{\perp}$ the factorization of $\bar{\pi}$ via $S^{\perp}$. The uniqueness of $\iota^{\perp}$ implies that $\iota^{\perp}=\bar{\iota}^{\perp} \circ \psi$. Hence, they have same image in $S^{\perp}, \iota^{\perp}(\Gamma)=\bar{\iota}^{\perp}(\bar{\Gamma})=\Gamma^{\perp}$, and project onto the same curve $\widetilde{\Gamma} \subset \widetilde{S}$. Furthermore, $\psi$ and $\tau^{\perp}$ being equivariant morphisms, we can push down $\psi: \Gamma \rightarrow \bar{\Gamma}$ to an identity between their quotients, $\Gamma / \tau_{\Gamma} \cong \mathbb{P}^{1} \rightrightarrows \mathbb{P}^{1} \cong \bar{\Gamma} / \tau_{\bar{\Gamma}}$, as well as $\bar{\iota}^{\perp}$ to a morphism $\widetilde{\iota}: \mathbb{P}^{1} \rightarrow \widetilde{\Gamma}$ (of same degree as $\tau^{\perp}: \bar{\Gamma} \rightarrow \Gamma^{\perp}$ ), as shown hereafter:

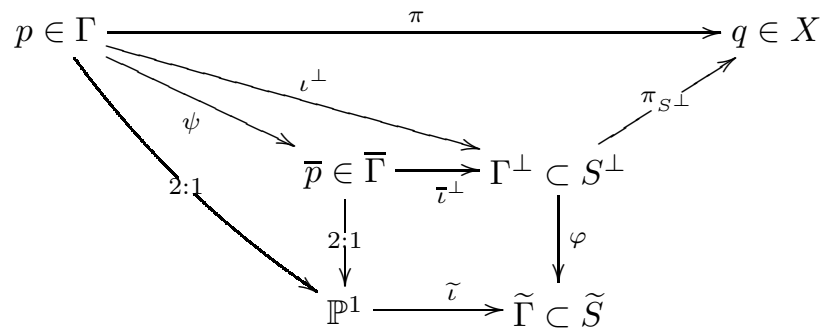

Taking the fiber product of $\widetilde{\iota}: \mathbb{P}^{1} \rightarrow \widetilde{\Gamma}$ and $\varphi: \Gamma^{\perp} \rightarrow \widetilde{\Gamma}$, say $\Gamma^{\star}$, we then factorize $\bar{\iota}^{\perp}$ in the above diagram, through a birational morphism $\bar{\Gamma} \rightarrow \Gamma^{\star}$ as follows:

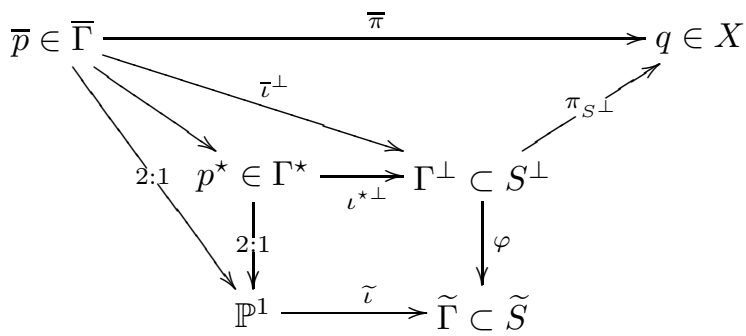

where $p^{\star} \in \Gamma^{\star}$ is the image of $\bar{p} \in \bar{\Gamma}$. Furthermore, since $\bar{p}$ is smooth and the unique pre-image of $p^{\star}$, we deduce that the latter morphism factorizes via the desingularization of $\Gamma^{\star}$ at the unibranch point $p^{\star}$. We will therefore assume till the end of the proof, that $\Gamma^{\star}$ is indeed smooth at $p^{\star}$. On the other hand, the degree-2 projection $\left(\bar{\Gamma} \rightarrow \mathbb{P}^{1}\right.$ is ramified at $\bar{p}$, hence) $\Gamma^{\star} \rightarrow \mathbb{P}^{1}$ is ramified at $p^{\star}$. Then, applying 3.8. one immediately checks that the natural projection $\pi^{\star}:=\pi_{S^{\perp}} \circ \iota^{\star \perp}:\left(\Gamma^{\star}, p^{\star}\right) \rightarrow(X, q)$ is a hyperelliptic d-osculating cover, dominated by $\bar{\pi}$ (and $\pi$ as well). Thus, the latter $\pi^{\star}$ is the unique minimal-hyperelliptic d-osculating cover dominated by $\pi$

Remark 3.10.

The minimal-hyperelliptic d-osculating cover $\pi^{\star}$, explicitely constructed in the proof of 3.9., can not be recovered from $\widetilde{\Gamma}:=\varphi\left(\Gamma^{\perp}\right)$, unless $m:=\operatorname{deg}\left(\iota^{\perp}: \Gamma \rightarrow \Gamma^{\perp}\right)$ is equal to 1 . There exists indeed a $(m-1)$-dimensional family of (non-isomorphic) 
minimal-hyperelliptic d-osculating covers, with same image $\widetilde{\Gamma} \subset \widetilde{S}$, as shown hereafter. We will actually start in 3.11. from a minimal-hyperelliptic d-osculating cover $\pi$ (i.e.: identifying $\Gamma$ with $\Gamma^{\star}$ ), and give its complete factorization, in terms of the rational curve $\widetilde{\Gamma} \subset \widetilde{S}$.

\section{Corollary 3.11.}

Let $\pi:(\Gamma, p) \rightarrow(X, q)$ be a minimal-hyperelliptic d-osculating cover, equipped (3.8.) with $\iota^{\perp}: \Gamma \rightarrow \Gamma^{\perp}$, its equivariant factorization through $S^{\perp}$, as well as $\mathbb{P}^{1} \stackrel{j}{\rightarrow} \widetilde{\Gamma}$, the desingularization of the rational irreducible curve $\widetilde{\Gamma}:=\varphi\left(\Gamma^{\perp}\right)$. Then, there exist unique marked morphisms $\psi:(\Gamma, p) \rightarrow\left(\Gamma^{b}, p^{b}\right), \pi^{b}:\left(\Gamma^{b}, p^{b}\right) \rightarrow(X, q)$ and $\iota^{b \perp}:\left(\Gamma^{b}, p^{b}\right) \rightarrow\left(\Gamma^{\perp}, \iota^{\perp}(p)\right)$, such that (see the diagrams below):

(1) $\pi$ and $\iota^{\perp}$ factor as $\pi^{\mathrm{b}} \circ \psi$ and $\iota^{\mathrm{b} \perp} \circ \psi$, respectively;

(2) $\operatorname{deg}(\psi)=m:=\operatorname{deg}\left(\iota^{\perp}\right)$, and $\psi^{-1}\left(p^{b}\right)=\{p\} ;$

(3) $\pi^{b}$ is a minimal-hyperelliptic $d^{b}$-osculating cover, where $2 d-1=m\left(2 d^{b}-1\right)$;

(4) there exist a polynomial morphism $R:\left(\mathbb{P}^{1}, \infty\right) \stackrel{m: 1}{\longrightarrow}\left(\mathbb{P}^{1}, \infty\right)$ and a degree-2 projection $\left(\Gamma^{b}, p^{b}\right) \stackrel{f^{b}}{\rightarrow}\left(\mathbb{P}^{1}, \infty\right)$, such that $\Gamma$ is the fiber product of $R$ with $f^{b}$;

(5) the arithmetic geni of $\Gamma$ and $\Gamma^{b}$, say $g$ and $g^{b}$, satisfy $2 g+1=m\left(2 g^{b}+1\right)$.

(6) $\Gamma$ is isomorphic to $\Gamma^{\perp}$, if and only if, $m=1$ and $\widetilde{\Gamma}$ is isomorphic to $\mathbb{P}^{1}$.

Furthermore, the moduli space of degree-n minimal-hyperelliptic d-osculating covers, having same image $\widetilde{\Gamma} \subset \widetilde{S}$ as $\pi$, is birational to a $(m-1)$-dimensional linear space.

Proof. (1)-(2)-(3) Let $\Gamma^{b}$ denote the fiber product of $\Gamma^{\perp} \stackrel{\varphi}{\rightarrow} \widetilde{\Gamma}$ and $\mathbb{P}^{1} \stackrel{j}{\rightarrow} \widetilde{\Gamma}$, equipped with the corresponding birational morphism $\Gamma^{b} \stackrel{\iota^{b \perp}}{\rightarrow} \Gamma^{\perp}$ and degree-2 cover $\Gamma^{b} \stackrel{f^{b}}{\rightarrow} \mathbb{P}^{1}$. The equivariant morphism $\iota^{\perp}$ can be pushed down, as in 3.9., to $\mathbb{P}^{1} \stackrel{\widetilde{\iota}}{\rightarrow} \widetilde{\Gamma}$ and factors through $j$, say $\widetilde{\imath}=j \circ R$. Moreover, the latter morphisms satisfy $\varphi \circ \iota^{\perp}=\widetilde{\iota}=j \circ R$, implying the factorization through the fiber product $\Gamma^{b}$. In other words, there exists a degree- $m$ equivariant morphism $\Gamma \stackrel{\psi}{\rightarrow} \Gamma^{b}$ (i.e.: $\psi \circ \tau_{\Gamma}=$ $\tau_{\Gamma^{b}} \circ \psi$ ), such that $\iota^{\perp}=\iota^{b \perp} \circ \psi$, and with maximal ramification index at $p \in \Gamma$ (i.e.: $\psi^{-1}\left(p^{b}\right)=\{p\}$, the fiber of $\iota^{\perp}$ over $\iota^{\perp}(p)$ ). In particular $\Gamma^{b}$ is unibranch at $p^{b}$, and up to replacing $\left(\Gamma^{b}, p^{b}\right)$ by its desingularization at $p^{b}$, we can assume $\pi^{b}:=\pi_{S \perp} \circ \iota^{b \perp}:\left(\Gamma^{b}, p^{b}\right) \rightarrow(X, q)$ is a hyperelliptic cover. This construction is sketched in the diagrams below: 

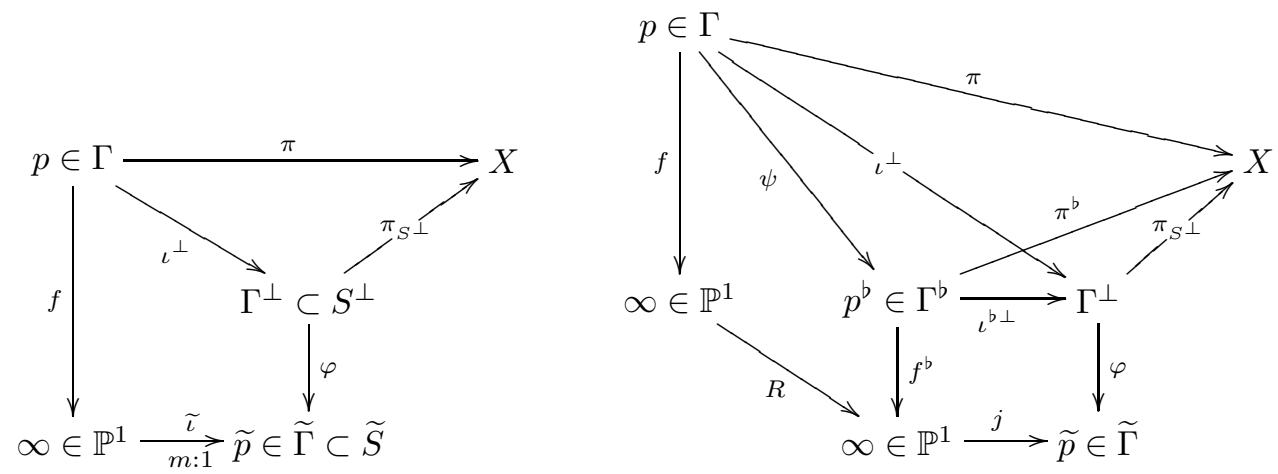

According to 3.8., the osculating order of $\pi^{b}($ 2.4. $(2))$, say $d^{b}$, satisfies $2 d^{b}$ $1=e^{*}\left(C_{o}\right) \cdot \iota^{b \perp}{ }_{*}\left(\Gamma^{b}\right)$, while $2 d-1=e^{*}\left(C_{o}\right) \cdot \iota^{\perp}{ }_{*}(\Gamma)$. On the other hand, the factorization $\iota^{\perp}=\iota^{b \perp} \circ \psi$ gives $\iota^{\perp}(\Gamma)=\iota^{b \perp}\left(\psi_{*}(\Gamma)\right)=\iota^{b \perp}\left(m \Gamma^{b}\right)$, and replacing in the former equality gives $2 d-1=m\left(2 d^{b}-1\right)$. Moreover, the minimal-hyperelliptic $d^{b}$-osculating cover dominated by $\pi^{b}$ (3.9.) has same image $\Gamma^{\perp}$ as $\pi^{b}$, hence, it must dominate the fiber product product of $\Gamma^{\perp} \stackrel{\varphi}{\rightarrow} \widetilde{\Gamma}$ and $\mathbb{P}^{1} \stackrel{j}{\rightarrow} \widetilde{\Gamma}$, and $\Gamma^{b}$ as well. In other words, $\pi^{b}$ is minimal-hyperelliptic.

(4) Recall that $\left(\Gamma^{b}, p^{b}\right) \stackrel{f^{b}}{\longrightarrow}\left(\mathbb{P}^{1}, \infty\right)$ is classically represented in affine coordinates, as the zero locus $\left\{y^{2}=P(x)\right\}$ projecting onto the first coordinate, for some degree$\left(2 g^{b}+1\right)$ polynomial $P(x), p^{b}$ being identified with the smooth Weierstrass point added at infinity. On the other hand, $\mathbb{P}^{1} \stackrel{R}{\rightarrow} \mathbb{P}^{1}$, the pushed down of $\Gamma \stackrel{\psi}{\rightarrow} \Gamma^{b}$ defined above, has maximal ramification index at $f(p) \in \mathbb{P}^{1}$ (i.e.: $f(p) \in \mathbb{P}^{1}$ is the unique pre-image of $\left.f^{b}\left(p^{b}\right) \in \mathbb{P}^{1}\right)$. Therefore, up to identifying the latter points with $\infty \in \mathbb{P}^{1}$, we may say that $\left(\mathbb{P}^{1}, \infty\right) \stackrel{R}{\rightarrow}\left(\mathbb{P}^{1}, \infty\right)$ is defined by a degree- $m$ polynomial $R(t)$. Taking the fiber product of $\Gamma^{b} \stackrel{f^{b}}{\longrightarrow} \mathbb{P}^{1}$ with $\mathbb{P}^{1} \stackrel{R}{\longrightarrow} \mathbb{P}^{1}$, amounts then to replacing $x$ by $R(t)$, giving the affine equation $\left\{y^{2}=P(R(t))\right\}$, where the composed polynomial $P(R(t))$ has odd degree equal to $\left(2 g^{b}+1\right) m$. Hence, the latter fiber product is a hyperelliptic curve, say $\Gamma_{R}$, of arithmetic genus $g_{R}$ such that $2 g_{R}+1=$ $m\left(2 g^{b}+1\right)$, equipped with a smooth Weierstrass point $p_{R} \in \Gamma_{R}$ and a marked projection $\left(\Gamma_{R}, p_{R}\right) \stackrel{m: 1}{\longrightarrow}\left(\Gamma^{b}, p^{b}\right)$, fitting in the following diagram:




We can also check that $p_{R} \in \Gamma_{R}$ is the unique pre-image of $p^{b} \in \Gamma^{b}$, i.e.: the ramification index of $\left(\Gamma_{R}, p_{R}\right) \stackrel{m: 1}{\longrightarrow}\left(\Gamma^{b}, p^{b}\right)$ at $p_{R}$ is equal to $m$. Hence, if $\kappa^{b}$ is the hyperelliptic $d^{b}$-osculating function for $\pi^{b}$, its inverse image gives a hyperelliptic $d$-osculating function for $\pi_{R}$. In other words, $\pi_{R}$ is a hyperelliptic $d$-osculating cover dominated by the minimal-hyperelliptic d-osculating cover $\pi$. Hence, they are isomorphic, implying that $\pi$ factors as $\pi^{b} \circ \psi, 2 g+1=m\left(2 g^{b}+1\right)$, and $\Gamma$ is the fiber product of $\mathbb{P}^{1} \stackrel{R}{\longrightarrow} \mathbb{P}^{1}$ and $\Gamma^{b} \stackrel{f^{b}}{\longrightarrow} \mathbb{P}^{1}$, as claimed.

(5) It follows from the latter constructions that $\Gamma$ is isomorphic to $\Gamma^{\perp}$, if and only if $j: \mathbb{P}^{1} \rightarrow \widetilde{\Gamma}$ is an isomorphism and $m=1$.

Consider at last, any other minimal-hyperelliptic d-osculating cover having same image $\widetilde{\Gamma} \subset \widetilde{S}$. The latter must also factor through the above minimal-hyperelliptic $d^{b}$-osculating cover $\pi^{b}$. We may replace then $R$ by any other degree- $m$ separable polynomial $P: \mathbb{P}^{1} \rightarrow \mathbb{P}^{1}$, and take its fiber product with $\Gamma^{b} \stackrel{f^{b}}{\longrightarrow} \mathbb{P}^{1}$, to produce the general degree-n minimal-hyperelliptic d-osculating cover having image $\widetilde{\Gamma}$. Up to isomorphism, they are parameterized by a $(m-1)$-dimensional linear space.

\section{The hyperelliptic $d$-OSCUlating COVERS AS DIVISORS OF A SURFACE}

4.1. The next step concerns studying the $\tau^{\perp}$-invariant irreducible curve $\Gamma^{\perp} \subset S^{\perp}$, associated in 3. to any hyperelliptic cover $\pi$. We calculate its linear equivalence class, in terms of the numerical invariants of $\pi$, and dress the basic relations between them. We also prove, whenever $\boldsymbol{p}:=\operatorname{char}(\mathbb{K}) \geq 3$, the supplementary bound $2 g+1 \leq \boldsymbol{p}(2 d-1)$ (4.4.(1) \& (6)). We end up giving a numerical characterization for $\pi$ to be minimal-hyperelliptic (4.6.).

\section{Definition 4.2.}

For any $i=0, . ., 3$, the intersection number between the divisors $\iota^{\perp}(\Gamma)$ and $r_{i}^{\perp}$ will be denoted by $\gamma_{i}$, and the corresponding vector $\gamma=\left(\gamma_{i}\right) \in \mathbb{N}^{4}$ called the type of $\pi$. Furthermore, for any $\mu=\left(\mu_{i}\right) \in \mathbb{N}^{4}, \mu^{(1)}$ and $\mu^{(2)}$ will denote, respectively:

$$
\mu^{(1)}:=\sum_{i=0}^{3} \mu_{i} \quad \text { and } \quad \mu^{(2)}:=\sum_{i=0}^{3} \mu_{i}^{2} .
$$

\section{Lemma 4.3.}

Let $(\Gamma, p) \stackrel{\pi}{\rightarrow}(X, q)$ be a degree-n hyperelliptic d-osculating cover, of type $\gamma$ and ramification index $\rho$ at $p$. Consider its unique equivariant factorization through $S^{\perp}, \iota^{\perp}: \Gamma \rightarrow \Gamma^{\perp}$, and let $m$ denote its degree and $\iota:=e \circ \iota^{\perp}$ its composition with the blowing up $S^{\perp} \stackrel{e}{\rightarrow} S$. Then :

(1) $\iota_{*}(\Gamma)$ is equal to $m . \iota(\Gamma)$ and linearly equivalent to $n C_{o}+(2 d-1) S_{o}$;

(2) $\iota_{*}(\Gamma)$ is unibranch, and transverse to the fiber $S_{o}:=\pi_{S}^{*}(q)$, at $s_{o}=\iota(p)$;

(3) $\rho$ is odd, bounded by $2 d-1$ and equal to the multiplicity of $\iota_{*}(\Gamma)$ at $s_{o}$;

(4) the degree $m$ divides $n, 2 d-1$ and $\rho$, as well as $\gamma_{i}$, for any $i \in\{0, . ., 3\}$;

(5) $\gamma_{o}+1 \equiv \gamma_{1} \equiv \gamma_{2} \equiv \gamma_{3} \equiv n(\bmod .2)$;

(6) $\iota^{\perp}(\Gamma)$ is linearly equivalent to $e^{*}\left(n C_{o}+(2 d-1) S_{o}\right)-\rho s_{o}^{\perp}-\sum_{i=0}^{3} \gamma_{i} r_{i}^{\perp}$.

Proof. (1) Checking that $\iota_{*}(\Gamma)$ is numerically equivalent to $n C_{o}+(2 d-1) S_{o}$ amounts to proving that the intersections numbers $\iota_{*}(\Gamma) \cdot S_{o}$ and $\iota_{*}(\Gamma) \cdot C_{o}$ are 
equal to $n$ and $2 d-1$. The latter numbers are equal, respectively, to the degree of $\pi: \Gamma \rightarrow X$ and the degree of $\iota^{*}\left(C_{o}\right)=(2 d-1) p$, hence the result. Finally, since $\iota_{*}(\Gamma)$ and $C_{o}$ only intersect at $s_{o} \in S_{o}$, we also obtain their linear equivalence.

(2) \& (3) Let $\kappa: \Gamma \rightarrow \mathbb{P}^{1}$ be the hyperelliptic d-osculating function associated to $\pi$, uniquely characterized by properties 2.6.(1),(2)\&(3), and $U \subset X$ a symmetric neighborhood of $q:=\pi(p)$. Recall that $\kappa+\pi^{*}\left(z^{-1}\right)$ is $\tau_{\Gamma}$-anti-invariant and well defined over $\pi^{-1}(U)$, where it has a (unique) pole of order $2 d-1$ at $p$. Studying its trace with respect to $\pi$ we can deduce that $\rho$ must be odd and bounded by $2 d-1$.

On the other hand, let $\left(\iota_{*}(\Gamma), S_{o}\right)_{s_{o}}$ and $\left(\iota_{*}(\Gamma), C_{o}\right)_{s_{o}}$ denote the intersection multiplicities at $s_{o}$, between $\iota_{*}(\Gamma)$ and the curves $S_{o}$ and $C_{o}$. They are respectively equal, via the projection formula for $\iota$, to $\rho$ and $2 d-1$. At last, since $\iota_{*}(\Gamma)$ is unibranch at $s_{o}$ and $\left(\iota_{*}(\Gamma), S_{o}\right)_{s_{o}}=\rho \leq 2 d-1=\left(\iota_{*}(\Gamma), C_{o}\right)_{s_{o}}$, we immediately deduce that $\rho$ is the multiplicity of $\iota_{*}(\Gamma)$ at $s_{o}$ (and $S_{o}$ is transverse to $\iota_{*}(\Gamma)$ at $s_{o}$ ).

(4) By definition of $m$, we clearly have $\iota_{*}(\Gamma)=m . \iota(\Gamma)$, while $\left\{\rho, \gamma_{i}, i=0, . ., 3\right\}$ are the multiplicities of $\iota_{*}(\Gamma)$ at different points of $S$. Hence, $m$ divides $n$ and $2 d-1$, as well as all integers $\left\{\rho, \gamma_{i}, i=0, . ., 3\right\}$.

(5) For any $i=0, . ., 3$, the strict transform of the fiber $S_{i}:=\pi_{S}^{-1}\left(\omega_{i}\right)$, by the monoidal transformation $e: S^{\perp} \rightarrow S$, is a $\tau^{\perp}$-invariant curve, equal to $S_{i}^{\perp}:=$ $e^{*}\left(S_{i}\right)-s_{i}^{\perp}-r_{i}^{\perp}$, but also to $\varphi^{*}\left(\widetilde{S}_{i}\right)$, where $\widetilde{S}_{i}:=\varphi\left(S_{i}^{\perp}\right)$. Hence, the intersection number $\iota^{\perp}(\Gamma) \cdot S_{i}^{\perp}$ is equal to the even integer

$$
\iota^{\perp}(\Gamma) \cdot S_{i}^{\perp}=\iota^{\perp}(\Gamma) \cdot \varphi^{*}\left(\widetilde{S}_{i}\right)=\varphi_{*}\left(\iota^{\perp}(\Gamma)\right) \cdot \widetilde{S}_{i}=2 \widetilde{\Gamma} \cdot \widetilde{S}_{i},
$$

implying that $n=\iota^{\perp}{ }_{*}(\Gamma) \cdot e^{*}\left(S_{i}\right)$ is congruent mod.2 to

$$
\iota^{\perp} *(\Gamma) \cdot S_{i}^{\perp}+\iota^{\perp} *(\Gamma) \cdot\left(s_{i}^{\perp}+r_{i}^{\perp}\right) \equiv \iota^{\perp} *(\Gamma) \cdot\left(s_{i}^{\perp}+r_{i}^{\perp}\right)(\bmod .2) .
$$

We also know, by definition, that $\gamma_{i}:=\iota^{\perp}{ }_{*}(\Gamma) \cdot r_{i}^{\perp}$, while $\iota^{\perp}{ }_{*}(\Gamma) \cdot s_{o}^{\perp}=\rho$, the multiplicity of $\iota_{*}(\Gamma)$ at $s_{o}$, and $\iota^{\perp} *(\Gamma) \cdot s_{i}^{\perp}=0$ if $i \neq 0$, because $s_{i} \notin \iota(\Gamma)$. Hence, $n$ is congruent mod.2, to $\rho+\gamma_{o} \equiv 1+\gamma_{o}(\bmod .2)$, as well as to $\gamma_{i}$, if $i \neq 0$.

(6) The Picard group $\operatorname{Pic}\left(S^{\perp}\right)$ is the direct sum of $e^{*}(\operatorname{Pic}(S))$ and the rank8 lattice generated by the exceptional curves $\left\{s_{i}^{\perp}, r_{i}^{\perp}, i=0, . ., 3\right\}$. In particular, knowing that $\iota_{*}(\Gamma)$ is linearly equivalent to $n C_{o}+(2 d-1) S_{o}$, and having already calculated $\iota^{\perp} *(\Gamma) \cdot s_{i}^{\perp}$ and $\iota^{\perp}(\Gamma) \cdot r_{i}^{\perp}$, for any $i=0, . ., 3$, we can finally check that $\iota^{\perp}{ }_{*}(\Gamma)$ is linearly equivalent to $e^{*}\left(n C_{o}+(2 d-1) S_{o}\right)-\rho s_{o}^{\perp}-\sum_{0}^{3} \gamma_{i} r_{i}^{\perp}$.

\section{Theorem 4.4 .}

Consider any hyperelliptic d-osculating cover $\pi:(\Gamma, p) \rightarrow(X, q)$, of degree $n$, type $\gamma$, arithmetic genus $g$ and ramification index $\rho$ at $p$. Let $m$ denote the degree of its canonical equivariant factorization $\iota^{\perp}: \Gamma \rightarrow \Gamma^{\perp} \subset S^{\perp}$, and $\widetilde{g}$ the arithmetic genus of the rational irreducible curve $\widetilde{\Gamma}:=\varphi\left(\Gamma^{\perp}\right)$. Then, the numerical invariants $\{n, d, g, \widetilde{g}, \rho, m, \gamma\}$ satisfy the following inequalities:

(1) $2 g+1 \leq \gamma^{(1)} \quad$;

(2) $4 m^{2} \widetilde{g}=(2 d-1)(2 n-2 m)+4 m^{2}-\rho^{2}-\gamma^{(2)}$ and $\gamma^{(2)} \leq 2(2 d-1)(n-m)+4 m^{2}-\rho^{2}$; 
(3) $(2 g+1)^{2} \leq 8(2 d-1)(n-m)+13 m^{2}-4 \rho^{2} \leq 8(2 d-1) n+(2 d-1)^{2} ;$

(4) $\rho=1$ implies $m=1$, as well as $(2 g+1)^{2} \leq 8(2 d-1)(n-1)+9$;

(5) if $\boldsymbol{p} \geq 3$, we must also have $\gamma^{(1)} \leq \boldsymbol{p}(2 d-1)$.

Proof. (1) For any $i=0, . ., 3$, the fiber of $\pi_{S^{\perp}}:=\pi_{S} \circ e: S^{\perp} \rightarrow X$ over the half-period $\omega_{i}$, decomposes as $s_{i}^{\perp}+r_{i}^{\perp}+S_{i}^{\perp}$, where $S_{i}^{\perp}$ is a $\tau^{\perp}$-invariant divisor and $s_{i}^{\perp}$ is disjoint with $\iota^{\perp} *(\Gamma)$, if $i \neq 0$, while $\iota^{\perp^{*}}\left(s_{i}^{\perp}\right)=\rho p$, by 4.3.(2). Hence, the divisor $R_{i}:=\iota^{\perp^{*}}\left(r_{i}^{\perp}\right)$ of $\Gamma$ is linearly equivalent to $R_{i} \equiv \pi^{-1}\left(\omega_{i}\right)-\left(n-\gamma_{i}\right) p$ (and also $\left.2 R_{i} \equiv 2 \gamma_{i} p\right)$. Recalling at last, that $\sum_{j=1}^{3} \omega_{j} \equiv 3 \omega_{o}$, and taking inverse image by $\pi$, we finally obtain that $\sum_{i=0}^{3} R_{i} \equiv \gamma^{(1)} p$. In other words, there exists a well defined meromorphic function, (i.e.: a morphism), from $\Gamma$ to $\mathbb{P}^{1}$, with a pole of (odd!) degree $\gamma^{(1)}$ at the Weierstrass point $p$. The latter can only happen (by the Riemann-Roch Theorem) if $2 g+1 \leq \gamma^{(1)}$, as asserted.

(2) The curve $\Gamma^{\perp}$ is $\tau^{\perp}$-invariant and linearly equivalent (4.3.(4)\&(6)) to:

$$
\Gamma^{\perp} \sim \frac{1}{m}\left(e^{*}\left(n C_{o}+(2 d-1) S_{o}\right)-\rho s_{o}^{\perp}-\sum_{i=0}^{3} \gamma_{i} r_{i}^{\perp}\right) .
$$

Recall also that $\widetilde{g} \geq 0$ and $\widetilde{K}$, the canonical divisor of $\widetilde{S}$, is linearly equivalent to $\varphi_{*}\left(e^{*}\left(-C_{0}\right)\right)\left([14 \oint 4.2 .(3))\right.$. Applying the projection formula for $S^{\perp} \stackrel{\varphi}{\rightarrow} \widetilde{S}$, to $\Gamma^{\perp}=\varphi^{*}(\widetilde{\Gamma})$, we obtain $0 \leq \widetilde{g}=\frac{1}{4 m^{2}}\left((2 d-1)(2 n-2 m)+4 m^{2}-\rho^{2}-\gamma^{(2)}\right)$, implying $\gamma^{(2)} \leq(2 d-1)(2 n-2 m)+4 m^{2}-\rho^{2}$, as claimed.

(3) \& (4) We start remarking that, for any $j=1,2,3,\left(\gamma_{o}-\gamma_{j}\right)$ is a non-zero multiple of $m$. Hence, $\sum_{i<j}\left(\gamma_{i}-\gamma_{j}\right)^{2} \geq 3 m^{2}$, and replacing in 4.4.(1) we get:

$$
(2 g+1)^{2} \leq\left(\gamma^{(1)}\right)^{2}=4 \gamma^{(2)}-\sum_{i<j}\left(\gamma_{i}-\gamma_{j}\right)^{2} \leq 4 \gamma^{(2)}-3 m^{2} .
$$

Taking into account 4.4.(3), we obtain the inequality 4.4.(4). At last, since $m$ divides $\rho$ (4.3.(4)), $\rho=1$ implies $m=1$. Replacing in 4.4.(3) gives us 4.4.(4).

(5) Finally, let us assume $\boldsymbol{p} \geq 3$ and denote by $C_{p}^{\perp} \subset S^{\perp}$ the unique $\tau^{\perp}$-invariant irreducible curve, linearly equivalent to $e^{*}\left(\boldsymbol{p} C_{o}\right)-\sum_{i=0}^{3} r_{i}^{\perp}$. In particular, it can not be equal to $\Gamma^{\perp}$, hence $C_{\boldsymbol{p}}^{\perp} \cdot \Gamma^{\perp}=\boldsymbol{p}(2 d-1)-\gamma^{(1)}$ must be non-negative.

\section{Corollary 4.5.}

Let $\pi: \Gamma \rightarrow X$ be a degree-n separable projection of a hyperelliptic curve onto the elliptic curve $X$, and let $g$ denote its arithmetic genus. Then, there exists a smooth Weierstrass point $p \in \Gamma$ such that $\pi:(\Gamma, p) \rightarrow(X, \pi(p))$ is a hyperelliptic $d$-osculating cover, non ramified at $p$, with d satisfying: $(2 d-1)(2 n-2) \geq g^{2}+g-2$.

Proof. Consider the global desingularization morphism $\bar{j}: \bar{\Gamma} \rightarrow \Gamma$, composed, either with $\pi$, or with the degree- 2 cover $\Gamma \rightarrow \Gamma / \tau_{\Gamma} \cong \mathbb{P}^{1}$. As a ramified cover of $X$ and $\mathbb{P}^{1}$, we deduce from the Hurwitz formula that $\bar{\Gamma}$ is a smooth hyperelliptic curve of positive genus, say $\bar{g}$, with $2 \bar{g}+2$ Weierstrass points, while $\bar{\pi}:=\pi \circ \bar{j}: \bar{\Gamma} \rightarrow X$ has, at most, $2 \bar{g}-2$ ramifications points. We can choose, therefore, a Weierstrass 
point $\bar{p} \in \bar{\Gamma}$, at which $\bar{\pi}$ is not ramified. In particular, its image $p:=\bar{j}(\bar{p}) \in \Gamma$ must be a unibranch point. On the other hand, since $\bar{\pi}$ is not ramified at $\bar{p}$ and factors through $\pi: \Gamma \rightarrow X$, we see that $\pi$ restricts to a local isomorphism between neighborhoods of $p \in \Gamma$ and $q:=\pi(p) \in X$ :

$$
\bar{\pi}: \bar{p} \in \bar{\Gamma} \stackrel{\bar{j}}{\rightarrow} p \in \Gamma \stackrel{\pi}{\rightarrow} q \in X
$$

Hence, $p$ is a smooth Weierstrass point of $\Gamma$, at which $\pi$ is not ramified, and $\pi:(\Gamma, p) \rightarrow(X, q)$ is a hyperelliptic d-osculating cover (2.4.(2)), for some integer $d \leq g$. Applying 4.4.(4), we obtain $(2 d-1)(2 n-2) \geq(g+2)(g-1)$ as claimed.

\section{Corollary 4.6.}

Let $\pi:(\Gamma, p) \rightarrow(X, q)$ be a hyperelliptic d-osculating cover of type $\gamma$ and arithmetic genus $g$. Then $2 g+1 \leq \gamma^{(1)}$, with equality if and only if $\pi$ is minimal-hyperelliptic.

Proof. Recall that $\pi$ dominates a unique minimal-hyperelliptic d-osculating (3.9.), say $\pi^{\star}$, factoring through the same curve $\Gamma^{\perp} \subset S^{\perp}$. Therefore, $\pi^{\star}$ has same type $\gamma$ as $\pi$, but a bigger arithmetic genus, say $g^{\star}$, satisfying $2 g+1 \leq 2 g^{\star}+1 \leq \gamma^{(1)}$ (4.4.(1)). Hence, it is certainly enough to assume $\pi$ is minimal-hyperelliptic and prove that $2 g+1 \geq \gamma^{(1)}$.

Recall also, that $\iota^{\perp}: \Gamma \rightarrow \Gamma^{\perp}$ has odd degree $m$ and factors through the cover $\pi^{b}:\left(\Gamma^{b}, p^{b}\right) \rightarrow(X, q)$, of type $\gamma^{b}$ and arithmetic genus $g^{b}$, such that $\gamma^{(1)}=m \gamma^{b(1)}$ and $2 g+1=m\left(2 g^{b}+1\right)$ (3.11. \& 4.3. (4)). Hence $2 g+1=m\left(2 g^{b}+1\right) \leq m \gamma^{b(1)}=$ $\gamma^{(1)}$, with equality if and only if $2 g^{b}+1=\gamma^{b(1)}$. We have thus reduced the problem, from $\pi$ to the minimal-hyperelliptic $\pi^{b}$. So let us suppose in the sequel that $m=1$, or in other words, that $(\Gamma, p)=\left(\Gamma^{b}, p^{b}\right)$. Let $\left(\Gamma^{\diamond}, p^{\diamond}\right)$ denote the fiber product of the marked morphisms $\left(\Gamma^{\perp}, \iota^{\perp}(p)\right) \stackrel{\varphi}{\longrightarrow}(\widetilde{\Gamma}, \widetilde{p})$ and $\left(\mathbb{P}^{1}, \infty\right) \stackrel{j}{\longrightarrow}(\widetilde{\Gamma}, \widetilde{p})($ 3.11. $)$. The marked curve $(\Gamma, p)=\left(\Gamma^{b}, p^{b}\right)$, is in fact the desingularization of $\Gamma^{\diamond}$ at its unibranch point $p^{\diamond}$ (3.11.), and fits in the following diagram:

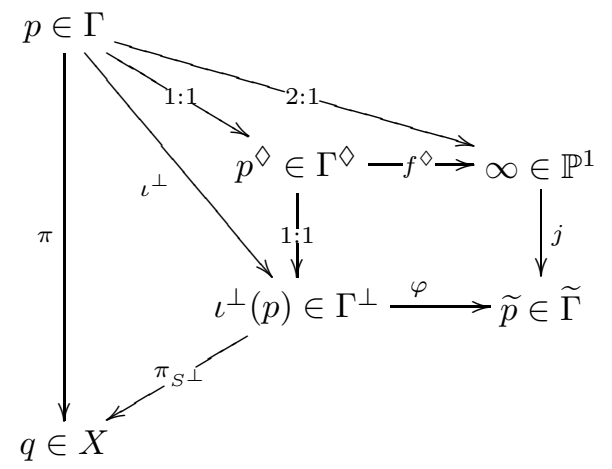

Let $\widetilde{g}, g^{\perp}, g^{\diamond}$ and $g$ denote the arithmetic geni of $\widetilde{\Gamma}, \Gamma^{\perp}, \Gamma^{\diamond}$ and $\Gamma$, respectively. Knowing the numerical equivalence class of $\Gamma^{\perp}$ we easily obtain (e.g.: 4.4.(2)):

$$
\widetilde{g}=\frac{1}{4}\left((2 d-1)(2 n-2)+4-\rho^{2}-\gamma^{(2)}\right) \quad \text { and } \quad g^{\perp}=2 \widetilde{g}+\frac{1}{2}\left(\rho-2+\gamma^{(1)}\right) .
$$

We can then deduce $g^{\diamond}$, arguing as follows (like in the proof of [14] 5 .8.(2)): since $\Gamma^{\perp} \stackrel{\varphi}{\longrightarrow} \widetilde{\Gamma}$ is a flat degree-2 morphism, and $\mathbb{P}^{1}$ has arithmetic genus $=0$, we 
must have the relation $g^{\perp}-g^{\diamond}=2(\widetilde{g}-0)=2 \widetilde{g}$. Hence, $g^{\diamond}=\frac{1}{2}\left(\rho-2+\gamma^{(1)}\right)$. We might as well argue that the desingularization morphism $\mathbb{P}^{1} \stackrel{j}{\rightarrow} \widetilde{\Gamma}$ is obtained by monoidal transformation $\widetilde{S}$ (i.e.: $j$ is the restriction of a finite sequence of monoidal transformations $\widetilde{S}^{\prime} \stackrel{j}{\longrightarrow} \widetilde{S}$ such that the strict transform of $\widetilde{\Gamma} \subset \widetilde{S}$ is isomorphic to $\mathbb{P}^{1}$ ), implying that $\Gamma^{\diamond}$ is contained in the fiber product of $S^{\perp} \stackrel{\varphi}{\longrightarrow} \widetilde{S}$ and $\widetilde{S}^{\prime} \stackrel{j}{\longrightarrow} \widetilde{S}$, for which we can calculate its canonical divisor. Applying the adjunction formula gives the above value of $g^{\diamond}$.

At last, composing $(\Gamma, p) \stackrel{1: 1}{\rightarrow}\left(\Gamma^{\diamond}, p^{\diamond}\right)$ with $\left(\Gamma^{\diamond}, p^{\diamond}\right) \stackrel{f^{\diamond}}{\longrightarrow}\left(\mathbb{P}^{1}, \infty\right)$, we get the degree 2 cover $f: \Gamma \stackrel{f}{\rightarrow} \mathbb{P}^{1}$, and a morphism $(f, \pi): \Gamma \rightarrow \Gamma_{f, \pi} \subset \mathbb{P}^{1} \times X$ as in 2.5., fitting in:

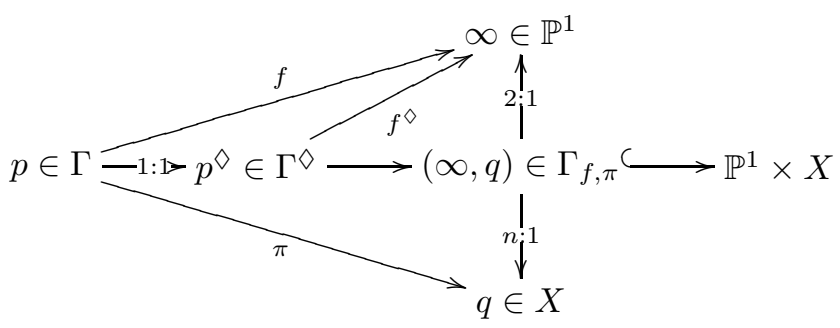

We have shown in the proof of $\mathbf{2 . 5}$.(3), that $\frac{1}{2}(\rho-1)$ consecutive monoidal transformations are necessary to desingularize $\Gamma_{f, \pi}$ at its unibranch point $(\infty, q)$, and each monoidal transformation lowers its arithmetic genus by 1 . On the other hand, since $(\Gamma, p)$ dominates $\left(\Gamma^{\diamond}, p^{\diamond}\right)$ and is smooth over $(\infty, q)$, we easily deduce that $g^{\diamond}-g \leq \frac{1}{2}(\rho-1)$. Hence $g^{\diamond}-\frac{1}{2}(\rho-1)=\frac{1}{2}\left(-1+\gamma^{(1)}\right) \leq g$.

\section{ON HYPERELLIPTIC $d$-OSCULATING COVERS OF ARBITRARY High GENUS}

5.1. - We will let $C_{o}^{\perp}$ and $C_{p}^{\perp}$ denote, hereafter, the strict transforms of $C_{o}$ and $C_{p}$ by $e: S^{\perp} \rightarrow S$ and $\widetilde{C}_{o}:=\varphi\left(C_{o}^{\perp}\right)$. Recall that to any hyperelliptic cover $\pi:(\Gamma, p) \rightarrow(X, q)$ we have uniquely associated a morphism $\iota^{\perp}: \Gamma \rightarrow \Gamma^{\perp} \subset S^{\perp}$, a rational irreducible curve $\widetilde{\Gamma}:=\varphi\left(\Gamma^{\perp}\right) \subset \widetilde{S}$ and a vector $(n, d, \rho, \gamma) \in \mathbb{N}^{* 3} \times \mathbb{N}^{4}$, satisfying the following restrictions (4.3. \& 4.4.) :

(1) $\rho$ is odd, bounded by $2 d-1$, and $\gamma_{o}+1 \equiv \gamma_{1} \equiv \gamma_{2} \equiv \gamma_{3} \equiv n(\bmod .2)$;

(2) if $\boldsymbol{p} \geq 3$, we must have $\gamma^{(1)} \leq \boldsymbol{p}(2 d-1)$.

Furthermore, $\pi$ can be canonically recovered from $\widetilde{\Gamma}:=\varphi\left(\Gamma^{\perp}\right)$ if, and only if, $\Gamma$ is birational to $\Gamma^{\perp}$, in which case:

(3) $\widetilde{\Gamma}$ has arithmetic genus $\widetilde{g}:=\frac{1}{4}\left((2 d-1)(2 n-2)+4-\rho^{2}-\gamma^{(2)}\right) \geq 0$;

(4) $\Gamma^{\perp}=\varphi^{*}(\widetilde{\Gamma})$ is linearly equivalent to $e^{*}\left(n C_{o}+(2 d-1) S_{o}\right)-\rho s_{o}{ }^{\perp}-\sum_{i=0}^{3} \gamma_{i} r_{i}{ }^{\perp}$;

(5) $\widetilde{\Gamma}$ intersects $\widetilde{s}_{o}:=\varphi\left(s_{o}^{\perp}\right)$, at a unique unibranch point, with multiplicity $\rho$;

(6) $\Gamma^{\perp}$ and $\widetilde{\Gamma}$ intersect $C_{o}^{\perp}$ and $\widetilde{C}_{o}$, (at most) at $p_{o}^{\perp}:=C_{o}^{\perp} \cap s_{o}^{\perp}$ and $\varphi\left(p_{o}^{\perp}\right)$, respectively, with multiplicities $2 d-1-\rho$ and $\frac{1}{2}(2 d-1-\rho)$.

\section{Definition 5.2.}

For any $(n, d, \rho, \gamma) \in \mathbb{N}^{* 3} \times \mathbb{N}^{4}$ satisfying 5.1.(1),(2)\&(3), we let $\Lambda(n, d, \rho, \gamma)$ denote the unique element of $\operatorname{Pic}(\widetilde{S})$ such that $\varphi^{*}(\Lambda(n, d, \rho, \gamma))$ is linearly equivalent 
to $e^{*}\left(n C_{o}+(2 d-1) S_{o}\right)-\rho s_{o}{ }^{\perp}-\sum_{i=0}^{3} \gamma_{i} r_{i}{ }^{\perp}$, and $M H_{X}(n, d, \rho, \gamma)$ denote the moduli space of degree-n minimal-hyperelliptic d-osculating covers of type $\gamma$, ramification index $\rho$ at their marked point, and birational to their canonical images in $S^{\perp}$.

\section{Proposition 5.3.}

Any $\pi \in M H_{X}(n, d, \rho, \gamma)$ can be canonically recovered from $\widetilde{\Gamma} \subset \widetilde{S}(\mathbf{3 . 1 1} .(2))$. Conversely, any rational irreducible curve $\widetilde{\Gamma} \subset \widetilde{S}$ satisfying properties 5.1.(1)-(6), gives rise to a unique element of $M H_{X}(n, d, \rho, \gamma)$.

Proof. Given $\widetilde{\Gamma} \subset \widetilde{S}$ satisfying 5.1.(1)-(6), we denote $\Gamma^{\perp}:=\varphi^{*}(\widetilde{\Gamma}) \subset S^{\perp}$ and consider the fiber product of $\left(\Gamma^{\perp}, p^{\perp}\right) \stackrel{\varphi}{\rightarrow}\left(\widetilde{\Gamma}, \varphi\left(p^{\perp}\right)\right)$ with the desingularization morphism $\left(\mathbb{P}^{1}, \infty\right) \stackrel{j}{\rightarrow}\left(\widetilde{\Gamma}, \varphi\left(p^{\perp}\right)\right)$, say $(\Gamma, p)$. Proceeding as in the proof of 3.11., for the construction of $\pi^{b}$, we can easily prove that the natural domination $(\Gamma, p) \rightarrow\left(\Gamma^{\perp}, p^{\perp}\right)$, composed with $\pi^{\perp}:\left(\Gamma^{\perp}, p^{\perp}\right) \rightarrow(X, q)$ is indeed the announced minimal-hyperelliptic d-osculating cover.

Studying $M H_{X}(n, d, \rho, \gamma)$ for a general vector $(n, d, \rho, \gamma)$, is a difficult and elusive problem. We will henceforth restrict to the simpler case where $\rho=1$ and $\widetilde{\Gamma}$ is isomorphic to $\mathbb{P}^{1}$. In other words, we will focus on degree- $n$ minimal-hyperelliptic $d$-osculating covers with $\rho=m=1$, and type $\gamma$ satisfying $\gamma^{(2)}=(2 d-1)(2 n-2)+3$ (as well as $\gamma^{(1)} \leq \boldsymbol{p}(2 d-1)$, if $\boldsymbol{p} \geq 3$ ).

Proposition 5.4. ([12 $\S 3.4)$

Any curve $\Gamma \subset S$ intersecting $C_{o}$ at a unique smooth point $p \in \Gamma$ is irreducible, unless $\boldsymbol{p} \geq 3$ and $C_{\boldsymbol{p}}$ is a component of $\Gamma$.

\section{Proposition 5.5.}

Let $\Gamma^{\perp} \subset S^{\perp}$ be a curve with no irreducible component in $\left\{r_{i}^{\perp}, i=0, . ., 3\right\}$, and intersecting $C_{o}^{\perp}$ (at most) at a unique smooth point $p^{\perp} \in \Gamma^{\perp}$. Then, $\Gamma^{\perp}$ is an irreducible curve, unless $\boldsymbol{p} \geq 3$ and $C_{p}^{\perp}$ is a component of $\Gamma^{\perp}$.

Proof. The properties satisfied by $\Gamma^{\perp}$ assure us that $\Gamma:=e_{*}\left(\Gamma^{\perp}\right)$, its direct image by $e: S^{\perp} \rightarrow S$, does not contain $C_{o}$, and that $\Gamma^{\perp}$ is the strict transform of $\Gamma$. We can also check, that $\Gamma$ is smooth at $p:=e\left(p^{\perp}\right)$ and $\Gamma \cap C_{o}=\{p\}$. It follows, by 5.4., that ( $\Gamma$, as well as its strict transform) $\Gamma^{\perp}$ is, either an irreducible curve, or $\boldsymbol{p} \geq 3$ and $C_{p}^{\perp}$ is a component of $\Gamma^{\perp}$.

Proposition 5.6. $(14 \S 6.2 . \&[10)$

Any $\alpha=\left(\alpha_{i}\right) \in \mathbb{N}^{4}$ such that $\alpha^{(2)}=2 a+1$ is odd (and $\alpha^{(1)} \leq \boldsymbol{p}$, whenever $\boldsymbol{p} \geq 3$ ), gives rise to an exceptional curve of the first kind $\widetilde{\Gamma}_{\alpha} \subset \widetilde{S}$. More precisely, let $k \in\{0,1,2,3\}$ denote the index satisfying $\alpha_{k}+1 \equiv \alpha_{j}$ (mod.2), for any $j \neq k$, and $S_{k}:=\pi_{S}^{-1}\left(\omega_{k}\right)$, then $\widetilde{\Gamma}_{\alpha}$ is a (-1)-curve and $\varphi^{*}\left(\widetilde{\Gamma}_{\alpha}\right) \subset S^{\perp}$ is the unique $\tau^{\perp}$ invariant irreducible curve linearly equivalent to $e^{*}\left(a C_{o}+S_{k}\right)-s_{k}^{\perp}-\sum_{i=0}^{3} \alpha_{i} r_{i}^{\perp}$.

Proof. Let $\Lambda$ denote the unique numerical equivalence class of $\widetilde{S}$ satisfying $\varphi^{*}(\Lambda)=e^{*}\left(a C_{o}+S_{k}\right)-s_{k}^{\perp}-\sum_{i=0}^{3} \alpha_{i} r_{i}^{\perp}$. It has self-intersection $\Lambda \cdot \Lambda=-1$, and $\Lambda \cdot \widetilde{K}=-1$ as well, hence, $h^{o}\left(\widetilde{S}, O_{\widetilde{S}}(\Lambda)\right) \geq \chi\left(O_{\widetilde{S}}(\Lambda)\right)=1$, and there exists an 
effective divisor $\widetilde{\Gamma} \in|\Lambda|$. If $\boldsymbol{p}=0$, such a divisor $\widetilde{\Gamma}$ is known to be unique and irreducible ([14]§6.2.). Its proof takes in account that for any $m>1$ there is no irreducible curve in $S$, numerically equivalent to $m C_{o}$. However, when $\boldsymbol{p} \geq 3$ the latter property fails, due to the existence of $C_{p} \subset S$, implying that the intersection number $C_{\boldsymbol{p}} \cdot \Lambda=\boldsymbol{p}$ - $\alpha^{(1)}$ must be non-negative. Conversely, if $\alpha^{(1)} \leq \boldsymbol{p}, \Lambda$ intersects non-negatively $\widetilde{C}_{p}:=\varphi\left(C_{p}{ }^{\perp}\right.$ ), (as well as all other (-1) and (-2)-curves in $\widetilde{S})$, and M.Lahyane's irreducibility criterion for (-1)-classes applies to $\Lambda$ ([10]).

According to 5.6., any $\alpha \in \mathbb{N}^{4}$ such that $\alpha^{(2)}$ is odd (and $\alpha^{(1)} \leq \boldsymbol{p}$, if $\boldsymbol{p} \geq 3$ ), gives rise to an exceptional curve of the first kind $\widetilde{\Gamma}_{\alpha} \subset \widetilde{S}$. Conversely, we have the

\section{Corollary 5.7.}

Any irreducible curve in $\widetilde{S}$, with negative self-intersection, is either equal to some $\widetilde{\Gamma}_{\alpha}$ as above (5.6.), to $\widetilde{C}_{\boldsymbol{p}}$ if $\boldsymbol{p} \geq 3$, or belongs to the set $\left\{\widetilde{C}_{o}, \widetilde{s}_{i}, \widetilde{r}_{i}, i=0, . ., 3\right\}$.

Proof. The arithmetic genus of an arbitrary irreducible curve $\widetilde{\Gamma} \subset \widetilde{S}$ is nonnegative and equal to $\widetilde{g}:=1+\frac{1}{2}(\widetilde{\Gamma} \cdot \widetilde{\Gamma}+\widetilde{\Gamma} \cdot \widetilde{K}) \geq 0$, where $\widetilde{K}$ denotes the canonical divisor of $\widetilde{S}$. In particular $\widetilde{\Gamma} \cdot \widetilde{\Gamma}+\widetilde{\Gamma} \cdot \widetilde{K} \geq-2$. Moreover, since $\varphi^{*}(\widetilde{K})=e^{*}\left(-2 C_{o}\right)$ (cf. [14) and $C_{o}$ is nef, we immediately deduce that $\widetilde{\Gamma} \cdot \widetilde{K} \leq 0$. Hence, $\widetilde{\Gamma} \cdot \widetilde{\Gamma}<0$ implies, either $\widetilde{\Gamma} \cdot \widetilde{\Gamma}=-2$ and $\widetilde{\Gamma} \cdot \widetilde{K}=0$, or $\widetilde{\Gamma} \cdot \widetilde{\Gamma}=-1=\widetilde{\Gamma} \cdot \widetilde{K}$. It follows, in any case, that $\widetilde{g}=0$, hence $\widetilde{\Gamma}$ is isomorphic to $\mathbb{P}^{1}$. If $\widetilde{\Gamma} \cdot \widetilde{\Gamma}=-1=\widetilde{\Gamma} \cdot \widetilde{K}$, one can easily check, via the projection formulae for $S^{\perp} \stackrel{\varphi}{\rightarrow} \widetilde{S}$ and $S^{\perp} \stackrel{e}{\rightarrow} S$, that $\Gamma^{\perp}:=\varphi^{*}(\widetilde{\Gamma})$ is a $\tau^{\perp}$-invariant divisor in $S^{\perp}$ and its projection in $S, \Gamma:=e_{*}\left(\Gamma^{\perp}\right)$, satisfies:

$$
\Gamma \cdot C_{o}=e_{*}\left(\Gamma^{\perp}\right) \cdot C_{o}=\Gamma^{\perp} \cdot e^{*}\left(C_{o}\right)=-\frac{1}{2} \Gamma^{\perp} \cdot e^{*}\left(-2 C_{o}\right)=-\frac{1}{2} \Gamma^{\perp} \cdot \varphi^{*}(\widetilde{K})=-\widetilde{\Gamma} \cdot \widetilde{K}=1 .
$$

It immediately follows that $\Gamma$ (as well as $\Gamma^{\perp}$ ) is irreducible. Otherwise it would break as a sum of two divisors exchanged by $\tau: S \rightarrow S$, in which case the above intersection number $\Gamma \cdot C_{o}$ should have been even. In other words, $\Gamma$ is an irreducible $\tau$-invariant curve, intersecting $C_{o}$ at $s_{k}$, for a unique $k \in\{0,1,2,3\}$. Hence, $\Gamma$ is linearly equivalent to $a C_{o}+S_{k}$, for some $a \in \mathbb{N}$.

Recall also that $\Gamma^{\perp} \cdot\left(C_{o}^{\perp}+\sum_{i=0}^{3} s_{i}^{\perp}\right)=\Gamma^{\perp} \cdot e^{*}\left(C_{o}\right)=1$, and let $\alpha=\left(\alpha_{i}\right)$ denote the vector of intersection numbers $\left(\Gamma^{\perp} \cdot r_{i}^{\perp}\right)$. Then, $\Gamma^{\perp}$ is linearly equivalent to $e^{*}\left(a C_{o}+S_{k}\right)-s_{k}^{\perp}-\sum_{i=0}^{3} \alpha_{i} r_{i}^{\perp}$, and intersecting with the numerically equivalent curves $\left\{S_{i}^{\perp}:=e^{*}\left(S_{i}\right)-s_{i}^{\perp}-r_{i}^{\perp}, i=0,1,2,3\right\}$ one easily finds out that $\alpha_{k}+1 \equiv$ $\alpha_{i}(\bmod .2)$, for any $i \neq k$. Moreover, its self-intersection is equal to

$$
2 a-1-\alpha^{(2)}=\Gamma^{\perp} \cdot \Gamma^{\perp}=\varphi^{*}(\widetilde{\Gamma}) \cdot \Gamma^{\perp}=\widetilde{\Gamma} \cdot \varphi_{*}\left(\Gamma^{\perp}\right)=2 \widetilde{\Gamma} \cdot \widetilde{\Gamma}=-2 .
$$

In other words, $2 a+1=\alpha^{(2)}$ and $\widetilde{\Gamma}=\widetilde{\Gamma}_{\alpha}$ (5.6.).

At last, let us suppose that $\widetilde{\Gamma} \cdot \widetilde{\Gamma}=-2$ and $\widetilde{\Gamma} \cdot \widetilde{K}=0$, but $\widetilde{\Gamma}$ does not belong to $\left\{\widetilde{s}_{i}, \widetilde{r}_{i}, i=0, . ., 3\right\}$. It then follows that $\Gamma^{\perp}:=\varphi^{*}(\widetilde{\Gamma})$ is a $\tau^{\perp}$-invariant divisor of $S^{\perp}$, of self-intersection $\Gamma^{\perp} \cdot \Gamma^{\perp}=-4$, equal to the strict transform of $\Gamma:=e\left(\Gamma^{\perp}\right) \subset S$. Therefore, it must be, either an irreducible degree- 2 cover of $\widetilde{\Gamma}$, or break as the sum of two copies of $\widetilde{\Gamma} \simeq \mathbb{P}^{1}$, interchanged by $\tau^{\perp}$. In the latter case, $\Gamma^{\perp}$ should be the strict transform of the divisor $\pi_{S}{ }^{-1}\left(q^{\prime}+[-1] q^{\prime}\right)$, for some $q^{\prime} \in X$, in which case 
HYPERELLIPTIC $d$-OSCULATING COVERS AND RATIONAL SURFACES

$\Gamma^{\perp} \cdot \Gamma^{\perp} \neq-4$. Hence, $\Gamma^{\perp}$ is indeed irreducible (and $\Gamma=e_{*}\left(\Gamma^{\perp}\right)$ as well). On the other hand, recalling that $\varphi^{*}(\widetilde{K})=e^{*}\left(-2 C_{o}\right)$ and $\varphi_{*}\left(\Gamma^{\perp}\right)=2 \widetilde{\Gamma}$, we obtain

$$
\Gamma \cdot\left(-2 C_{o}\right)=e_{*}\left(\Gamma^{\perp}\right) \cdot\left(-2 C_{o}\right)=\Gamma^{\perp} \cdot e^{*}\left(-2 C_{o}\right)=\Gamma^{\perp} \cdot \varphi^{*}(\widetilde{K})=2 \widetilde{\Gamma} \cdot \widetilde{K}=0,
$$

implying $\Gamma$ is numerically equivalent to a multiple of $C_{o}$ According to 3.5. this can only happen if $\Gamma=C_{o}$ and $\Gamma^{\perp}=C_{o}^{\perp}$, or $\boldsymbol{p} \geq 3, \Gamma=C_{\boldsymbol{p}}$ and $\Gamma^{\perp}=C_{\boldsymbol{p}}^{\perp}$.

\section{Lemma 5.8.}

Let $\Lambda:=\Lambda(n, d, 1, \gamma)$ be as in 5.2., $\widetilde{\Gamma}$ an arbitrary exceptional curve of the first kind on $\widetilde{S}$ and $\alpha \in \mathbb{N}^{4}$ the unique vector as in 5.6. such that $\widetilde{\Gamma}=\widetilde{\Gamma}_{\alpha}$ (5.7.). Then:

$$
4(2 d-1) \widetilde{\Gamma}_{\alpha} \cdot \Lambda=\left\{\begin{array}{l}
(\gamma-(2 d-1) \alpha)^{(2)}-(2 d-1)^{2}-3 \quad, \quad \text { if } \quad \Gamma_{\alpha} \cdot \widetilde{s}_{o}=1 \\
(\gamma-(2 d-1) \alpha)^{(2)}+2(2 d-1)-(2 d-1)^{2}-3 \quad \text { otherwise } .
\end{array}\right.
$$

Proof. Straightforward verification

For $\Lambda(n, d, 1, \gamma)$ to be nef, we must have $\Lambda(n, d, 1, \gamma) \cdot \widetilde{\Gamma}_{\alpha} \geq 0$, for any $\alpha$ as above. On the other hand, minimizing their value is tantamount (5.8.) to minimizing the norm of $\gamma-(2 d-1) \alpha$. In order to do it we make the following definitions.

\section{Definition 5.9.}

(1) Given $(n, d, \gamma) \in \mathbb{N}^{*} \times \mathbb{N}^{*} \times \mathbb{N}^{4}$ satisfying $\gamma_{o}+1 \equiv \gamma_{j}(\bmod .2), \forall j=1,2,3$, as well as $\gamma^{(2)}=(2 d-1)(2 n-2)+3$, we let $\gamma=(2 d-1) \mu+2 \varepsilon$ be the unique decomposition, with $\mu \in \mathbb{N}^{4}$ having same parity as $\gamma$, and $\varepsilon \in \mathbb{Z}^{4}$ such that $\max \left\{\left|\varepsilon_{i}\right|\right\} \leq d-1$. We will also assume, here and henceforth, that $\gamma^{(1)}=(2 d-1) \mu^{(1)}+2 \varepsilon^{(1)} \leq \boldsymbol{p}(2 d-1)$, whenever $\boldsymbol{p} \geq 3$.

(2) We define ${ }^{\natural} \mu=\left({ }^{\natural} \mu_{i}\right) \in \mathbb{N}^{4}$ in order to have $\left({ }^{\natural} \mu_{i}-\mu_{i}\right) \varepsilon_{i}=\left|\varepsilon_{i}\right|, \forall i=0, \cdots, 3$ :

$$
{ }^{\natural} \mu_{i}=\mu_{i}+1 \quad \text { if } \quad \varepsilon_{i} \geq 0 \quad \text { or } \quad{ }^{\natural} \mu_{i}=\mu_{i}-1 \quad \text { if } \varepsilon_{i}<0
$$

(3) At last, we choose two indices $i_{o} \neq j_{o}$, where $\left|\varepsilon_{i}\right|$ attains its two maximal values, and let ${ }^{b} \mu=\left({ }^{b} \mu_{i}\right) \in \mathbb{N}^{4}$ be such that for all $i \in\{0,1,2,3\}$ :

$$
{ }^{\mathrm{b}} \mu_{i}={ }^{\natural} \mu_{i} \quad \text { if } \quad i \in\left\{i_{o}, j_{o}\right\} \quad \text { or } \quad{ }^{\mathrm{b}} \mu_{i}=\mu_{i} \quad \text { if } i \notin\left\{i_{o}, j_{o}\right\}
$$

Remark 5.10.

The vector ${ }^{b} \mu$ may not be uniquely defined by 5.9.(3). It should also be clear that $\mu \equiv \gamma(\bmod .2)$, and $4 \varepsilon^{(2)} \equiv 3(\bmod .(2 d-1))$. Conversely, we have the

\section{Proposition 5.11.}

Given any $n \in \mathbb{N}^{*}$ and $\gamma=(2 d-1) \mu+2 \varepsilon$, with $\mu \in \mathbb{N}^{4}$ and $\varepsilon \in \mathbb{Z}^{4}$, such that:

$$
\begin{aligned}
& \mu_{o}+1 \equiv \mu_{1} \equiv \mu_{2} \equiv \mu_{3}(\bmod .2) \\
& 4 \varepsilon^{(2)} \equiv 3(\bmod .(2 d-1)) \quad \text { and }\left|\varepsilon_{i}\right| \leq d-1, \quad i=0, \cdots, 3, \\
& \gamma^{(2)}=(2 d-1)(2 n-2)+3,
\end{aligned}
$$$$
\text { (as well as } \quad \gamma^{(1)} \leq \boldsymbol{p}(2 d-1), \quad \text { if } \boldsymbol{p} \geq 3 \text { ), }
$$ 
the minimal value of $\Lambda(n, d, 1, \gamma) \cdot \widetilde{\Gamma}_{\alpha}$, taken amongst all $\alpha \in \mathbb{N}^{4}$ with $\alpha^{(2)}$ odd, is attained at $\alpha$ equal, either to $\mu$, to ${ }^{\natural} \mu$, or to ${ }^{b} \mu$.

\section{Corollary 5.12.}

The divisor $\Lambda(n, d, 1, \gamma)$ is nef if and only if the vector $2 \varepsilon=\gamma-(2 d-1) \mu \in \mathbb{Z}^{4}$ (5.9.), such that $4 \varepsilon^{(2)} \equiv 3(\bmod .(2 d-1))$ and $\max \left\{\left|\varepsilon_{i}\right|\right\} \leq d-1$, satisfies the supplementary conditions :

(1) $\varepsilon^{(2)} \geq d^{2}-d+1$

(2) $(2 d-1)\left({ }^{\natural} \mu-\mu\right) \cdot \varepsilon=(2 d-1)\left(\sum_{i=0}^{3}\left|\varepsilon_{i}\right|\right) \leq 3 d^{2}-3 d+\varepsilon^{(2)}$;

(3) $(2 d-1)\left({ }^{b} \mu-\mu\right) \cdot \varepsilon=\max \left\{\left|\varepsilon_{i}\right|+\left|\varepsilon_{j}\right|, \forall i \neq j,\right\} \leq d^{2}-1+\varepsilon^{(2)}$.

As we shall see, given any $n, d \in \mathbb{N}^{*}$, there exist types $\gamma=(2 d-1) \mu+2 \varepsilon \in \mathbb{N}^{4}$, such that $\gamma_{o}+1 \equiv \gamma_{1} \equiv \gamma_{2} \equiv \gamma_{3}(\bmod .2)$ and $\gamma^{(2)}=(2 n-2)(2 d-1)+3$, for which $\Lambda:=\Lambda(n, d, 1, \gamma)$ is, either nef or not. We will actually construct in 5.13. and 5.14., explicit examples where, either $\varepsilon$ satisfies 5.12.(1),(2) \&(3), hence $\Lambda$ is nef, or it does not satisfy $\mathbf{5 . 1 2}$.(1), hence $\Lambda$ is not nef. We actually conjecture that 5.13. exhausts all types such that $\gamma^{(2)}=(2 d-1)(2 n-2)+3$ and $\Lambda(n, d, 1, \gamma)$ is nef.

\section{Proposition 5.13}

Let us fix $d \geq 2, k \in\{0,1,2,3\}$, and $\mu \in \mathbb{N}^{4}$ such that $\mu_{o}+1 \equiv \mu_{j}(\bmod .2)$ (for $j=1,2,3)$. Pick any vector $2 \varepsilon=\left(2 \varepsilon_{i}\right) \in 2 \mathbb{Z}^{4}$, satisfying $(\forall i=0, \ldots, 3)$ :

either $\quad\left|2 \varepsilon_{i}\right|=(2 d-2)\left(1-\delta_{i, k}\right), \quad$ or $\left\{\begin{array}{l}\left|2 \varepsilon_{i}\right|=d-(-1)^{\delta_{i, k}} \quad \text { if } \quad d \quad \text { is odd, } \\ \left|2 \varepsilon_{i}\right|=d-2 \delta_{i, k}\end{array} \quad\right.$ if $d \quad$ is even.

Then, for $n$ satisfying $\gamma^{(2)}=(2 d-1)(2 n-2)+3$, and assuming $\gamma:=(2 d-1) \mu+2 \varepsilon$ belongs to $\mathbb{N}^{4}$ (as well as $\gamma^{(1)} \leq \boldsymbol{p}(2 d-1)$, if $\boldsymbol{p} \geq 3$ ), the divisor $\Lambda(n, d, 1, \gamma)$ is nef.

Proof. One only needs to check (straightforward verification!), that any such $\varepsilon$ satisfies 5.12.(1),(2) \& (3).

\section{Proposition 5.14.}

Let us fix $d \geq 3$ and $\mu \in \mathbb{N}^{4}$ such that $\mu_{o}+1 \equiv \mu_{j}(\bmod .2)$ (for $\left.j=1,2,3\right)$, and let $k$ denote the residue (mod.4) of $d+1$. Choose any integer vector $\varepsilon \in \mathbb{Z}^{4}$ subject to the conditions

$$
4 \varepsilon^{(2)}=3+(2 d-1)(d-2+k) \quad \text { and } \quad \gamma:=(2 d-1) \mu+2 \varepsilon \in \mathbb{N}^{4},
$$

and let $n$ satisfy $\gamma^{(2)}=(2 d-1)(2 n-2)+3$. Then $\Lambda(n, d, 1, \gamma)$ is not nef.

Proof. Take any vector $\varepsilon \in \mathbb{Z}^{4}$ satisfying $\varepsilon^{(2)}=8 h^{2}+3(2 k-3) h+k^{2}-3 k+3$. A straightforward verification shows that $\varepsilon_{i}^{2} \leq \varepsilon^{(2)}<(2 d-1)^{2}, \forall i=0, . ., 3$ and $4 \varepsilon^{(2)}=3+(2 d-1)(d-2+k)$. In particular, $4 \varepsilon^{(2)}<3+(2 d-1)^{2}=4 d^{2}-4 d+4$, hence $\varepsilon$ does not satisfy property $\mathbf{5 . 1 2}$.(1). Therefore, choosing any $\mu \in \mathbb{N}^{4}$ such that $\mu_{o}+1 \equiv \mu_{j}(\bmod .2)$ (for $\left.j=1,2,3\right)$, and defining $\gamma \in \mathbb{N}^{4}$ and $n \in \mathbb{N}$ by $\gamma:=(2 d-1) \mu+2 \varepsilon$ and $\gamma^{(2)}=(2 d-1)(2 n-2)+3$, respectively, the corresponding 
divisor $\Lambda(n, d, 1, \gamma)$ is not nef.

\section{Lemma 5.15.}

Let $(n, d, \gamma) \in \mathbb{N}^{*} \times \mathbb{N}^{*} \times \mathbb{N}^{4}$ be such that $d \geq 2, \gamma^{(2)}=(2 d-1)(2 n-2)+3$ and $\Lambda(n, d, 1, \gamma)$ is nef. Then, for any $j=1,2,3$, there exists at most one exceptional curve of the first kind $\widetilde{\Gamma} \subset \widetilde{S}$, such that $\widetilde{\Gamma} \cdot \Lambda(n, d, 1, \gamma)=0$ and $\widetilde{\Gamma} \cdot \widetilde{s}_{j}=1$. In particular, the sum of the latter exceptional curves, denoted by $\widetilde{Z}(n, d, 1, \gamma)$, is a reduced divisor with (at most) three irreducible components.

Proof. Straightforward verification again!.

\section{Remark 5.16.}

According to Brian Harbourne's results on anticanonical rational surfaces (cf. 6]), for any nef divisor $D \in \operatorname{Pic}(\widetilde{S})$, such that $-\widetilde{K} \cdot D \geq 2$, the complete linear system $|D|$ is base point free and $\operatorname{dim}|D|=\frac{1}{2} D \cdot(D-\widetilde{K})$. The following result is in order.

\section{Lemma 5.17.}

Let $(n, d, \gamma) \in \mathbb{N}^{*} \times \mathbb{N}^{*} \times \mathbb{N}^{4}$ be such that $d \geq 2, \gamma^{(2)}=(2 d-1)(2 n-2)+3$, and let $\Lambda$ and $\widetilde{Z}$ denote, respectively, $\Lambda(n, d, 1, \gamma)$ and $\widetilde{Z}(n, d, 1, \gamma)$, the divisors defined in 5.15.. Then, $\Lambda$ nef implies:

(1) $\Lambda-\widetilde{C}_{o}-\sum_{j=1}^{3} \widetilde{s}_{j}-\widetilde{Z}$ is nef;

(2) $\left|\Lambda-\widetilde{C}_{o}-\sum_{j=1}^{3} \widetilde{s}_{j}-\widetilde{Z}\right|$ is base point free;

(3) $\left|\Lambda-\widetilde{C}_{o}\right|=\sum_{j=1}^{3} \widetilde{s}_{j}+\widetilde{Z}+\left|\Lambda-\widetilde{C}_{o}-\sum_{j=1}^{3} \widetilde{s}_{j}-\widetilde{Z}\right|$;

(4) $\operatorname{dim}|\Lambda|=2 d-2, \quad \operatorname{dim}\left|\Lambda-\widetilde{C}_{o}\right|=d-2 \quad$ and $\quad h^{1}\left(\widetilde{S}, O_{\widetilde{S}}\left(\Lambda-\widetilde{C}_{o}\right)\right)=0$.

\section{Definition 5.18.}

Let $\widetilde{p}_{o} \in \widetilde{S}$ denote the unique point of intersection $\left\{\widetilde{p}_{o}\right\}:=\widetilde{C}_{o} \cap \widetilde{s}_{o}$ and consider any divisor $\Lambda:=\Lambda(n, d, 1, \gamma)$ as in 5.15.. We define the following subsets of $|\Lambda|$ :

$$
\begin{aligned}
& |\Lambda|_{\widetilde{C}_{o}, \widetilde{p}_{o}}:=\left\{D \in|\Lambda|, \quad D \cap \widetilde{C}_{o}=\left\{\widetilde{p}_{o}\right\} \quad \text { or } \quad \widetilde{C}_{o} \subset D\right\} ; \\
& |\Lambda|_{\widetilde{C}_{o}, \widetilde{p}_{o}}^{\widetilde{s}_{o}}:=|\Lambda|_{\widetilde{C}_{o}, \widetilde{p}_{o}} \cap\left(\widetilde{s}_{o}+\left|\Lambda-\widetilde{s}_{o}\right|\right) .
\end{aligned}
$$

\section{Proposition 5.19.}

If $\Lambda:=\Lambda(n, d, 1, \gamma)$ is nef, then:

(1) $|\Lambda|_{\widetilde{C}_{o}, \widetilde{p}_{o}}$ is a $(d-1)$-dimensional subspace of $|\Lambda|$;

(2) $\widetilde{C}_{o}+\left|\Lambda-\widetilde{C}_{o}\right|$ and $|\Lambda|_{\widetilde{C}_{o}, \widetilde{p}_{o}}^{\widetilde{s}_{o}}$ are two different hyperplanes of $|\Lambda|_{\widetilde{C}_{o}, \widetilde{p}_{o}}$;

(3) any element $\widetilde{\Gamma} \in|\Lambda|_{\widetilde{C}_{o}, \widetilde{p}_{o}}$, in the complement of the latter hyperplanes, is a smooth integral divisor isomorphic to $\mathbb{P}^{1}$.

Proof. 
(1) According to 5.17.(4), we have $h^{1}\left(\widetilde{S}, O_{\widetilde{S}}\left(\Lambda-\widetilde{C}_{o}\right)\right)=0$. Hence, the exact sequence of $O_{\widetilde{S}}$-modules:

$$
0 \rightarrow O_{\widetilde{S}}\left(\Lambda-C_{o}\right) \rightarrow O_{\widetilde{S}}(\Lambda) \rightarrow O_{\widetilde{C}_{o}}(\Lambda) \rightarrow 0
$$

gives rise to the exact sequence

$$
0 \rightarrow H^{0}\left(\widetilde{S}, O_{\widetilde{S}}\left(\Lambda-\widetilde{C}_{o}\right)\right) \rightarrow H^{0}\left(\widetilde{S}, O_{\widetilde{S}}(\Lambda)\right) \rightarrow H^{0}\left(\widetilde{C}_{o}, O_{\widetilde{C}_{o}}(\Lambda)\right) \rightarrow 0 .
$$

Since $\operatorname{deg}\left(O_{\widetilde{C}_{o}}(\Lambda)\right)=d-1$, we can pick a section $f \in H^{0}\left(\widetilde{C}_{o}, O_{\widetilde{C}_{o}}(\Lambda)\right)$ which only vanishes at $\widetilde{p}_{o}$ (i.e.: with zero divisor $\left.(f)_{o}=(d-1) \widetilde{p}_{o}\right)$, as well as a preimage of $f$, say $v \in H^{0}\left(\widetilde{S}, O_{\widetilde{S}}(\Lambda)\right)$, such that its zero divisor $\widetilde{D}:=(v)_{o} \in|\Lambda|$ only intersects $\widetilde{C}_{o}$ at $\widetilde{p}_{o}$ (i.e.: $\widetilde{D} \cap \widetilde{C}_{o}=\left\{\widetilde{p}_{o}\right\}$ ). Any other section of $O_{\widetilde{S}}(\Lambda)$, satisfying the same property as $v$, is obtained by adding the image of an arbitrary element of $H^{0}\left(\widetilde{S}, O_{\widetilde{S}}\left(\Lambda-\widetilde{C}_{o}\right)\right)$. In other words $|\Lambda|_{\widetilde{C}_{o}, \widetilde{p}_{o}} \subset|\Lambda|$ is the $(d-1)$-dimensional subspace generated by $\widetilde{D}$ and $\widetilde{C}_{o}+\left|\Lambda-\widetilde{C}_{o}\right|$.

(2) On the other hand, according to 5.17.(2)\&(3), there exists $\widetilde{D}^{\prime} \in\left|\Lambda-\widetilde{C}_{o}\right|$ avoiding $\widetilde{p}_{o}$, in which case $\widetilde{C}_{o}+\widetilde{D}^{\prime} \in|\Lambda|$ is smooth at $\widetilde{p}_{o}$. Up to replacing the former divisor $\widetilde{D} \in|\Lambda|$, by the generic element of the pencil generated by $\widetilde{D}$ and $\left(\widetilde{C}_{o}+\widetilde{D}^{\prime}\right)$, we can assume hereafter $\widetilde{D}$ smooth and tangent to $\widetilde{C}_{o}$ at $\widetilde{p}_{o}$. In particular, for any $\widetilde{D}^{\prime \prime} \in\left|\Lambda-\widetilde{C}_{o}\right|$, either $\widetilde{p}_{o} \notin \widetilde{D}^{\prime \prime}$ and $\widetilde{C}_{o}+\widetilde{D}^{\prime \prime}$ is also smooth and tangent to $C_{o}$ at $\widetilde{p}_{o}$, or $\widetilde{p}_{o} \in \widetilde{D}^{\prime \prime}$ and $\widetilde{C}_{o}+\widetilde{D}^{\prime \prime}$ is singular at $\widetilde{p}_{o}$. In both cases, all but one element of the pencil generated by $\widetilde{D}$ and $\widetilde{C}_{o}+\widetilde{D}^{\prime \prime}$ is smooth and tangent to $C_{o}$ at $\widetilde{p}_{o}$. Therefore, such a generic element is transverse at $\widetilde{p}_{o}$ to $\widetilde{s}_{o}$, and can not contain $\widetilde{s}_{o}$ as an irreducible component. At last, since $\Lambda \cdot \widetilde{s}_{o}=1$, the unique singular element of the latter pencils must belong to $\widetilde{s}_{o}+\left|\Lambda-\widetilde{s}_{o}\right|$. Hence, $|\Lambda|_{\widetilde{C}_{o}, \widetilde{p}_{o}}^{\widetilde{s}_{o}}$ and $\widetilde{C}_{o}+\left|\Lambda-\widetilde{C}_{o}\right|$ are indeed distinct hyperplanes of $|\Lambda|_{\widetilde{C}_{o}, \widetilde{p}_{o}}$.

(3) Any $\widetilde{\Gamma} \in|\Lambda|_{\widetilde{C}_{o}, \widetilde{p}_{o}}$, in the complement of the latter hyperplanes, has arithmetic genus 0 . Let us also prove its irreducibility. We start remarking that $\widetilde{\Gamma}$ can only intersect $\widetilde{C}_{o}$ at $\widetilde{p}_{o}$, and does not contain $\widetilde{C}_{o}$ nor $\widetilde{s}_{i},(i=0,1,2,3)$, as an irreducible component. Hence, its inverse image $\Gamma^{\perp}:=\varphi^{*}(\widetilde{\Gamma}) \subset S^{\perp}$ is linearly equivalent to $e^{*}\left(n C_{o}+S_{o}\right)-s_{o}^{\perp}-\sum_{i=o}^{3} \gamma_{i} r_{i}^{\perp}$, and neither $C_{o}^{\perp}$, nor $s_{i}^{\perp}(\forall i=0, \ldots, 3)$, is an irreducible component of $\Gamma^{\perp}$. In order to check that $\Gamma^{\perp}$ (hence $\widetilde{\Gamma}$ ) is an irreducible curve, by means of $\mathbf{5 . 5}$., we still need to show that $r_{i}^{\perp} \nsubseteq \Gamma^{\perp}, \forall i=0, \ldots, 3$. Otherwise $\Gamma^{\perp}$ would have an irreducible component $\bar{\Gamma}^{\perp} \subset S^{\perp}$, linearly equivalent to $e^{*}\left(n C_{o}+S_{o}\right)-s_{o}^{\perp}-\sum_{i=o}^{3} \bar{\gamma}_{i} r_{i}^{\perp}$, for some type $\bar{\gamma}$ strictly bigger than $\gamma$, implying that $\varphi\left(\bar{\Gamma}^{\perp}\right) \subset \widetilde{S}$ has a negative arithmetic genus. Contradiction.! In case $\boldsymbol{p} \geq 3$, an analogous line of reasoning shows that $\Gamma^{\perp}$ can not contain $C_{p}^{\perp}$ as an irreducible component and 5.5. still applies.

Recalling that $M H_{X}(n, d, 1, \gamma)$ is birationally isomorphic to $|\Lambda(n, d, 1, \gamma)|_{\widetilde{C}_{o}, \widetilde{p}_{o}}$ (5.3.), we deduce the:

Corollary 5.20.

For any $(n, \mu) \in \mathbb{N}^{*} \times \mathbb{N}^{4}$ satisfying $\mu_{o}+1 \equiv \mu_{1} \equiv \mu_{2} \equiv \mu_{3}(\bmod .2)$ and $\mu^{(2)}=2 n+1$, 
(and $\mu^{(1)} \leq \boldsymbol{p}$, if $\boldsymbol{p} \geq 3$ ), we let $\pi_{\mu}$ denote the minimal-hyperelliptic 1-osculating cover associated to the exceptional curve $\widetilde{\Gamma}_{\mu} \subset \widetilde{S}$ (cf. 5.6. \& [14] $\$ 6.2$.). Then, $|\Lambda(n, d, 1, \gamma)|=\left\{\widetilde{\Gamma}_{\mu}\right\}$ and $M H_{X}(n, 1,1, \mu)$ reduces to $\left\{\pi_{\mu}\right\}$.

More generally, for any $(n, d, \gamma) \in \mathbb{N}^{*} \times \mathbb{N}^{*} \times \mathbb{N}^{4}$ such that:

(1) $\gamma_{o}+1 \equiv \gamma_{1} \equiv \gamma_{2} \equiv \gamma_{3}(\bmod .2) \quad\left(\right.$ and $\gamma^{(1)} \leq \boldsymbol{p}$, if $\boldsymbol{p} \geq 3$ ),

(2) $d \geq 2$ and $\gamma^{(2)}=(2 d-1)(2 n-2)+3$,

(3) $\Lambda(n, d, 1, \gamma)$ is nef,

the moduli space $M H_{X}(n, d, 1, \gamma)$ is birational to $|\Lambda(n, d, 1, \gamma)|_{\widetilde{C}_{o}, \widetilde{p}_{o}}$.

In particular, $\operatorname{dim}\left(M H_{X}(n, d, 1, \gamma)\right)=d-1$, for any $(n, d, \gamma)$ as in $\mathbf{5 . 1 3 .}$

At last, we propose a less conceptual but more geometrical construction of $M H_{X}(n, d, 1, \gamma)$. We will construct $d$ effective divisors $\left\{G^{\perp}, F_{j}^{\perp}, j=0, . ., d-2\right\}$ of $S^{\perp}$, with birational models given by explicit equations in $\mathbb{P}^{1} \times X$, which generate all $M H_{X}(n, d, 1, \gamma)$. Hence, any element of $M H_{X}(n, d, 1, \gamma)$ is birational to the zero set of a linear combination of $d$ specific degree- $n$ polynomials with coefficients in $K(X)$, the field of meromorphic functions on $X$.

\section{Theorem 5.21.}

For any $(n, d, \gamma) \in \mathbb{N}^{*} \times \mathbb{N}^{*} \times \mathbb{N}^{4}$ as in 5.13., $\left|e^{*}\left(n C_{o}+(2 d-1) S_{o}\right)-s_{o}^{\perp}-\sum_{i} \gamma_{i} r_{i}^{\perp}\right|$ contains a (d-1)-dimensional subspace with a generic element, say $\Gamma^{\perp}$, satisfying:

(1) $\Gamma^{\perp}$ is a $\tau^{\perp}$-invariant smooth irreducible curve of genus $g:=\frac{1}{2}\left(-1+\gamma^{(1)}\right)$;

(2) $\Gamma^{\perp}$ can only intersect $C_{o}^{\perp}$ at $p_{o}^{\perp}:=C_{o}^{\perp} \cap s_{o}^{\perp}$;

(3) $\varphi\left(\Gamma^{\perp}\right) \subset \widetilde{S}$ is isomorphic to $\mathbb{P}^{1}$.

\section{Corollary 5.22.}

Given $(n, d, \gamma) \in \mathbb{N}^{*} \times \mathbb{N}^{*} \times \mathbb{N}^{4}$ as above, the moduli space $M H_{X}(n, d, 1, \gamma)($ 5.2.) has dimension $d-1$, and its generic element is smooth of genus $g:=\frac{1}{2}\left(-1+\gamma^{(1)}\right)$.

\section{Proof of Theorem 5.21..}

We will only work out the case $\gamma:=(2 d-1) \mu+2 \varepsilon$, with $\varepsilon=(0, d-1, d-1, d-1)$.

For any other choice of $\varepsilon$, the corresponding proof runs along the same lines and will be skipped. In our case, the arithmetic genus $g$ and the degree $n$ satisfy:

$2 g+1=(2 d-1) \mu^{(1)}+6(d-1) \quad$ and $\quad 2 n=(2 d-1) \mu^{(2)}+4(d-1)\left(\mu_{1}+\mu_{2}+\mu_{3}\right)+6 d-7$.

Consider $\bar{\mu}:=\mu+(1,1,1,1), \mu^{\prime}:=\mu+(0,2,1,1), \mu^{\prime \prime}=\mu+(0,0,1,1)$, and let $\bar{Z}^{\perp}, Z^{\prime \perp}, Z^{\prime \prime} \subset S^{\perp}$ denote the unique $\tau^{\perp}$-invariant curves linearly equivalent to:

1) $\bar{Z}^{\perp} \sim e^{*}\left(\bar{m} C_{o}+S_{o}\right)-s_{o}^{\perp}-\sum_{i} \bar{\mu}_{i} r_{i}^{\perp}$, where $2 \bar{m}+1=\bar{\mu}^{(2)}$;

2) $Z^{\prime \perp} \sim e^{*}\left(m^{\prime} C_{o}+S_{1}\right)-s_{1}^{\perp}-\sum_{i} \mu_{i}^{\prime} r_{i}^{\perp}$, where $2 m^{\prime}+1=\mu^{\prime(2)}$;

3) $Z^{\prime \prime} \perp e^{*}\left(m^{\prime \prime} C_{o}+S_{1}\right)-s_{1}^{\perp}-\sum_{i} \mu_{i}^{\prime \prime} r_{i}^{\perp}$, where $2 m^{\prime \prime}+1=\mu^{\prime \prime(2)}$. 
Moreover, if $\mu_{o} \neq 0$ we choose $\underline{\mu}=\mu+(-1,1,1,1)$ and $2 \underline{m}+1=\underline{\mu}^{(2)}$, and let $\underline{Z}^{\perp} \subset S^{\perp}$ denote the unique $\tau^{\perp-i n v a r i a n t}$ curve $\underline{Z}^{\perp} \sim e^{*}\left(\underline{m} C_{o}+S_{o}\right)-s_{o}^{\perp}-\sum_{i} \underline{\mu}_{i} r_{i}^{\perp}$.

However, if $\mu_{o}=0$ we will simply put $\underline{Z}^{\perp}:=\bar{Z}^{\perp}+2 r_{o}^{\perp}$, so that in both cases, the divisors $D_{0}^{\perp}:=\bar{Z}^{\perp}+\underline{Z}^{\perp}+2 s_{0}^{\perp}$ and $D_{1}^{\perp}:=Z^{\prime \perp}+Z^{\prime \prime}+2 s_{1}^{\perp}$ will be linearly equivalent. Let us also define,

$$
\begin{aligned}
\mu_{(1)}:=\mu^{\prime \prime} & =\mu+(0,0,1,1), \\
\mu_{(2)} & :=\mu+(0,1,0,1), \\
\mu_{(3)} & :=\mu+(0,1,1,0),
\end{aligned}
$$

and let $Z_{(k)}^{\perp}(k=1,2,3)$ be the $\tau^{\perp}$-invariant curve of $S^{\perp}$, linearly equivalent to $e^{*}\left(m_{(k)} C_{o}+S_{k}\right)-s_{k}^{\perp}-\sum_{i} \mu_{(k) i} r_{i}^{\perp}$, where $2 m_{(k)}+1=\sum_{i} \mu_{(k) i}^{2}$.

At last, consider $Z^{\perp} \sim e^{*}\left(m C_{o}+S_{o}\right)-s_{o}^{\perp}-\sum_{i} \mu_{i} r_{i}^{\perp}$, where $2 m+1=\sum_{i} \mu_{i}^{2}$ (5.2.). Let $\Lambda \in \operatorname{Pic}(\widetilde{S})$ denote the unique class such that $\left|e^{*}\left(n C_{o}+(2 d-1) S_{o}\right)-s_{o}^{\perp}-\sum_{i} \gamma_{i} r_{i}^{\perp}\right|=$ $\left|\varphi^{*}(\Lambda)\right|$. The $(d-1)$-dimensional subspace of $\left|\varphi^{*}(\Lambda)\right|$ we are looking for, will be made of all above curves. We first remark the following facts :

a) we can check via the adjunction formula, that the divisors $\varphi^{*}(\Lambda)$ and $\Lambda$ have arithmetic genus $g:=\frac{1}{2}\left(-1+\gamma^{(1)}\right)$ and 0 , respectively, and that $\varphi^{*}(|\Lambda|)$ is equal to $\left|\varphi^{*}(\Lambda)\right|^{\tau^{\perp}}$, the sub-space of $\tau^{\perp}$-invariant elements of $\left|\varphi^{*}(\Lambda)\right|$;

b) the $d$ - 1 divisors

$$
F_{j}^{\perp}:=C_{o}^{\perp}+\sum_{k=1}^{3}\left(Z_{(k)}^{\perp}+2 s_{k}^{\perp}\right)+j D_{o}^{\perp}+(d-2-j) D_{1}^{\perp}, \quad j=0, \ldots, d-2,
$$

as well as

$$
G^{\perp}:=Z^{\perp}+(d-1) D_{o}^{\perp}
$$

are $\tau^{\perp}$-invariant, belong to $\left|\varphi^{*}(\Lambda)\right|$ and have $p_{o}^{\perp}:=C_{o}^{\perp} \cap s_{o}^{\perp}$ as their unique common point;

c) the curve $F_{o}^{\perp}$ is smooth at $p_{o}^{\perp}$, while any other $F_{j}^{\perp}$ has multiplicity $1<2 j+1<2 d$ at $p_{o}^{\perp}$. In particular, they span a $(d-2)$-dimensional subspace of $\left|\varphi^{*}(\Lambda)\right|$, having a generic element smooth and transverse to $s_{o}^{\perp}$ at $p_{o}^{\perp}$;

d) the curve $G^{\perp}$ has multiplicity $2 d$ at $p_{o}^{\perp}$, and no common irreducible component with any $F_{j}^{\perp}(\forall j=0, \ldots, d-2)$, implying that $\left\langle G^{\perp}, F_{j}^{\perp}, j=0, . ., d-2\right\rangle$, the $(d-1)$ dimensional subspace they span in $\left|\varphi^{*}(\Lambda)\right|$, is component-free;

e) any irreducible curve $\Gamma^{\perp} \in\left\langle G^{\perp}, F_{j}^{\perp}, j=0, . ., d-2\right\rangle$ projects onto a smooth irreducible curve (isomorphic to $\mathbb{P}^{1}$ ). In particular $\Gamma^{\perp}$ must be smooth outside $\cup_{i=0}^{3} r_{i}^{\perp}$.

f) the curves $G^{\perp}$ and $F_{o}^{\perp}$ have no common point on any $r_{i}^{\perp}(i=0, . ., 3)$, implying that $\Gamma^{\perp}$, the generic element of $\left\langle G^{\perp}, F_{j}^{\perp}, j=0, . ., d-2\right\rangle$, is smooth at any point of $\cup_{i=0}^{3} r_{i}^{\perp}$ and satisfies the announced properties, i.e.: 
(1) $\Gamma^{\perp}$ is $\tau^{\perp}$-invariant, smooth and satisfies the irreducibility criterion $\mathbf{5 . 5}$;

(2) $p_{o}^{\perp}$ is the unique base point of the linear system and $\Gamma^{\perp} \cap C_{o}^{\perp}=\left\{p_{o}^{\perp}\right\}$;

(3) its image $\varphi\left(\Gamma^{\perp}\right) \subset \widetilde{S}$ is irreducible, linearly equivalent to $\Lambda(n, d, 1, \gamma)$ and of arithmetic genus $\frac{1}{4}\left((2 d-1)(2 n-2)+3-\gamma^{(2)}\right)=0$; hence, isomorphic to $\mathbb{P}^{1}$.

Proof of Corollary 5.22.

The degree-2 projection $\varphi: \Gamma^{\perp} \longrightarrow \varphi\left(\Gamma^{\perp}\right)$ is ramified at $p_{o}^{\perp}$ and $\varphi\left(\Gamma^{\perp}\right)$ is isomorphic to $\mathbb{P}^{1}$. Moreover, $\Gamma^{\perp}$ is a smooth irreducible curve linearly equivalent to $\varphi^{*}(\Lambda(n, d, 1, \gamma))$, of arithmetic genus $g:=\frac{1}{2}\left(\gamma^{(1)}-1\right)$.

In other words, the natural projection $\left(\Gamma^{\perp}, p_{o}^{\perp}\right) \subset\left(S^{\perp}, p_{o}^{\perp}\right) \stackrel{\pi_{S \perp}}{\longrightarrow}(X, q)$ is a smooth degree-n minimal-hyperelliptic d-osculating cover of type $\gamma$, and genus $g$, such that $(2 n-2)(2 d-1)+3=\gamma^{(2)}$ and $2 g+1=\gamma^{(1)}$.

\section{REFERENCES}

[1] Airault H., McKean H.P., Moser J., Rational and elliptic solutions of the Korteweg-deVries equation and a related many body problem, Comm. Pure Appl. Math., 30(1977), pp.95-148.

[2] Akhmetshin A.A., Krichever I.M. and Vol'vovskii Y.S., Elliptic families of solutions of the Kadomtsev-Petviashvili equation, and the field analog of the elliptic Calogero-Moser system, Funct. Anal. and its Appl., $36 n^{\circ} 4$ (2002), pp.253-266.

[3] Dubrovin B.A., Novikov S.P., A periodicity problem for the Korteweg-deVries-SturmLiouville equations. Their connection with algebraic geometry, Doklady Acad. Nauk SSSR, $219 n^{\circ}$ (1974), pp.19-22. .

[4] Flédrich P., Paires 3-tangentielles hyperelliptiques et solutions doublement périodiques en $t$ de l'équation de Korteweg-de Vries, Thèse Université d'Artois (Pôle de Lens), Déc. 2003.

[5] Flédrich P. \& Treibich A., Hyperelliptic osculating covers and KdV solutions periodic in t, I.M.R.N. 5 (2006), pp.1-17, Article ID 73476.

[6] Harbourne B., Anticanonical rational surfaces, Trans. A.M.S., $349 n^{\circ} 3$ (1997), pp.1191-1208.

[7] Hartshorne R., Algebraic Geometry, Grad.Texts in Math.52, Springer-Verlag (1977).

[8] Its A.R. \& Matveev V., Hill's operator with a finite number of lacunae and multisoliton solutions of the Korteweg-de Vries equation, Teoret. Mat. Fiz 23 (1975), pp.51-67.

[9] Krichever I.M., Elliptic solutions of the KP equation and integrable systems of particles, Funct. Anal., 14, 4 (1980), pp.45-54.

[10] Lahyane M., Irreducibility of the (-1)-classes on smooth rational surfaces, Proc.A.M.S., 133 $n^{\circ} 8$ (2005), pp.2219-2224.

[11] Smirnov A.O., Solutions of the KdV equation, elliptic in t, Teor. Mat.Fiz. $100 n^{\circ} 2$ (1994).

[12] Treibich A., Matrix elliptic solitons, Duke Math.J., $90 n^{\circ} 3$ (1997), pp.523-547.

[13] Treibich A., Revêtements hyperelliptiques d-osculateurs et solitons elliptiques de la hiérarchie $K d V$, C.R. Acad. Sci. Paris, Série I 345 (2007), pp.213-218.

[14] Treibich A. \& Verdier J.-L., Solitons Elliptiques, Progress in Math., 88. (app.by J.Oesterlé); The Grothendieck Festschrift. Ed. Birkhäuser (1990), pp.437-479. 